\title{
Theory of Optical Transitions in $\pi$-Conjugated Macrocycles
}

\author{
Max Marcus ${ }^{1,2}$, Jaymee Coonjobeeharry ${ }^{3}$, and William Barford ${ }^{1}$ \\ ${ }^{1}$ Department of Chemistry, Physical and Theoretical Chemistry Laboratory, \\ University of Oxford, Oxford, OX1 3QZ, United Kingdom \\ ${ }^{2}$ Magdalen College, University of Oxford, \\ Oxford, OX1 4AU, United Kingdom \\ ${ }^{3}$ Hertford College, University of Oxford, \\ Oxford, OX1 3BW, United Kingdom
}




\begin{abstract}
We describe a theoretical and computational investigation of the optical properties of $\pi$-conjugated macrocycles. Since the low-energy excitations of these systems are Frenkel excitons that couple to high-frequency dispersionless phonons, we employ the quantized Frenkel-Holstein model and solve it via the DMRG method. First we consider optical emission from perfectly circular systems. Owing to optical selection rules, such systems radiate via two mechanisms: (i) within the Condon approximation, by thermally induced emission from the optically allowed $j= \pm 1$ states and (ii) beyond the Condon approximation, by emission from the $j=0$ state via coupling with a totally non-symmetric phonon (namely, the Herzberg-Teller effect). Using perturbation theory, we derive an expression for the HerzbergTeller correction and show via DMRG calculations that this expression soon fails as $\hbar \omega / J$ and the size of the macrocycle increases. Next, we consider the role of broken symmetry caused by torsional disorder. In this case the quantum number $j$ no longer labels eigenstates of angular momentum, but instead labels localized local exciton groundstates (LEGSs) or quasi-extended states (QEESs). As for linear polymers, LEGSs define chromophores, with the higher energy QEESs being extended over numerous LEGSs. Within the Condon approximation (i.e., neglecting the Herzberg-Teller correction) we show that increased disorder increases the emissive optical intensity, because all the LEGSs are optically active. We next consider the combined role of broken symmetry and curvature, by explicitly evaluating the Herzberg-Teller correction in disordered systems via the DMRG method. The HerzbergTeller correction is most evident in the emission intensity ratio, $I_{00} / I_{01}$. In the Condon approximation $I_{00} / I_{01}$ is a constant function of curvature, whereas in practice it vanishes for closed rings and only approaches a constant in the limit of vanishing curvature. We calculate the optical spectra of a model system, cyclo-poly(para-phenylene ethynylene), for different amounts of torsional disorder within and beyond the Condon approximation. We show how broken symmetry and the Herzberg-Teller effect explain the spectral features. The Herzberg-Teller correction to the $0-1$ emission vibronic peak is always significant. Finally, we note the qualitative similarities between the optical properties of conformationally disordered linear polymers and macrocycles in the limit of sufficiently large disorder, because in both cases they are determined by the optical properties of curved chromophores.
\end{abstract}




\section{INTRODUCTION}

Conjugated polymers have attracted increased interest over the past decades after the discovery of their semiconducting and electroluminescent ${ }^{1-4}$ properties. Macrocycles form a specific class of conjugated polymers having a circular $\pi$-electron system that can have a diameter of up to several nanometers ${ }^{5-8}$. The most striking difference from linear conjugated polymers is the cyclic $\pi$-electron system, giving rise to different optical and electronic phenomena $^{9-20}$. These optical properties are widely exploited in nature, for instance in the macrocyclic system of porphyrin and its derivatives used in chlorophyll and related lightharvesting compounds ${ }^{21,22}$. Certain macrocycles, poly(para-phenylene) in particular, also form topologically the shortest carbon nanotubes and are therefore interesting compounds in which to study the basic features of nanotubes ${ }^{23}$. It is particularly their optical properties, however, which make this class of conjugated macrocycles interesting for possible technological applications. These applications include novel organic electronics, such as molecular switches. While linear conjugated polymers are widely investigated, macrocyles, due to their only recent synthetic availability, remain open for theoretical studies.

There are important similarities and differences between the electronic properties of linear polymers and macrocycles. The similarities are first, that the low-energy excited states of both systems are Frenkel excitons; second, the Frenkel exciton couples to local nondispersive high energy phonons (associated with the $\mathrm{C}-\mathrm{C}$ bond stretch) and to low energy torsional modes; and third, torsional degrees of freedom cause conformational disorder. As a consequence, the excited states of both systems are conveniently described by the disordered Frenkel-Holstein model ${ }^{24,25}$.

The excited states of both systems are also subject to localization. There are two possible mechanisms of localization. First, excitons that couple to a set of harmonic oscillators becomes 'self-trapped', i.e., there is a local displacement of the oscillator that is proportional to the local exciton density. If the oscillators are treated classically, the non-linear feedback induced by the exciton-oscillator coupling self-localizes the exciton and 'spontaneously' breaks

the translational symmetry. This is a self-localized (or auto-localized) 'Landau polaron'. ${ }^{26,27}$ Recent work on the fully quantized Frenkel-Holstein model ${ }^{28,29}$, however, indicates that selflocalization is only expected in the adiabatic limit. High frequency $\mathrm{C}-\mathrm{C}$ bond oscillations do not cause Landau polarons and lower frequency torsional modes are unlikely to do so $^{29}$. 
We therefore do not consider this mechanism of localization in this paper. A second mechanism for exciton localization in disordered systems is Anderson localization ${ }^{30,31}$, causing the formation of 'Anderson polarons'. In this paper we treat Anderson localization as the cause of broken symmetry. We note, however, that self-localization via self-trapping is widely assumed in the literature to determine the emissive properties of macrocycles, for example in cyclo-poly(para-phenylenes) $)^{15,16}$ and in giant macrocycles with a limited number of conformational degrees of freedom ${ }^{17,18}$. Conversely, disorder-induced localization was assumed in cyclo-poly(thiophenes) ${ }^{20}$.

The obvious difference between linear polymers and macrocycles is their shape, in principle leading to quite different optical properties. Although there are significant differences in optical properties between ideal, ordered linear polymers and uniform macrocycles (in particular with regard to emission where non-Condon effects are important ${ }^{13,14}$ ), a key aim of this paper is to show that these differences become less significant when comparing conformationally disordered systems. This is because the photophysical properties of disordered systems are determined by the optical properties of curved chromophores ${ }^{32-34}$, which (as we show) do not necessarily span the entire system.

Thus, the goals of this paper are first, to describe how temperature and Herzberg-Teller effects determine the optical transitions in perfectly circular macrocycles; second, to describe how conformational disorder - leading to a broken circular symmetry - affects the absorption and emission spectra; third, to describe the role of curvature (which manifests itself spectroscopically partially via the Herzberg-Teller effect) on the emission spectra of disordered systems; and finally, to show how these effects explain experimental observations.

To address these issues we use the fully quantized disordered Frenkel-Holstein model, where the Frenkel exciton and local normal modes are all quantum variables. Although we are predominately interested in the Born-Oppenheimer regime (as this is generally applicable to conjugated macrocycles), we solve the model using the density matrix renormalization group (DMRG) method, which is accurate for all parameter regimes. Where appropriate, we compare DMRG results to predictions of the Born-Oppenheimer-Condon approximation.

Since, as already stated, the optical properties of a disordered macrocycle are determined by the optical properties of curved chromophores, the work reported here is related to the recent investigation of the effect of chain bending on the optical properties of conjugated polymers by Hestand and Spano ${ }^{32}$. Hestand and Spano report a strong dependence of the 


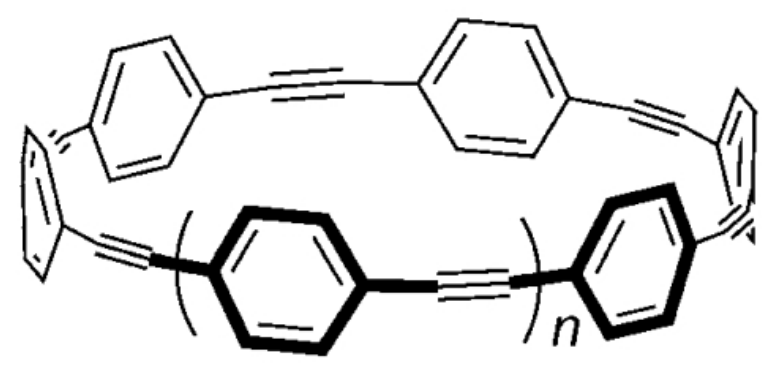

FIG. 1: Schematic representation of a CPPE macrocycle.

optical properties on the curvature of the chain in the anti-adiabatic limit, especially at low temperatures. As shown in Section V, we qualitatively reproduce their results.

The system principally investigated in this paper are cyclo-poly(para-phenyelene ethynylene) (CPPE) macrocycles, illustrated in Fig. 1; although in Section III model homomoiety systems are also studied. In Section II we introduce the Frenkel-Holstein model, briefly review DMRG, and review previously derived results on the theory of optical transitions within the Born-Oppenheimer-Condon approximation. Optical transitions in perfectly uniform circular systems are discussed in Section III, with a particular focus on the Herzberg-Teller corrections to the Condon approximation. The role of broken symmetry within and beyond the Condon approximation are described in Sections IV and V, respectively. In Section VI we describe the computed optical spectra of conformationally disordered CPPE macrocycles and interpret them in terms of the results of the previous sections. We summarize and conclude in Section VII. Appendix A contains a detailed derivation of the HerzbergTeller correction for conjugated macrocycles, while Appendix B and Appendix C explain the connection between the curvature of a chromophore and the Herzberg-Teller effect.

\section{MODEL AND METHODOLOGY}

\section{A. The Frenkel-Holstein Model}

The Frenkel-Holstein model is a coarse-grained model describing the delocalization of a Frenkel exciton and its coupling to a single local normal mode on each moiety. For the ethynylene moiety the normal mode is the symmetric vibration of the $\mathrm{C}-\mathrm{C}$ bond, while for the phenylene moiety the normal mode is the symmetric stretch associated with the 
aromatic to quinoid distortion. (The eigenvectors of these normal modes are given in the Supplementary Information of $\operatorname{ref}^{29}$.) The Frenkel-Holstein model has been widely used to describe the photophysical properties of J-aggregates ${ }^{35,36}$ and conjugated polymers ${ }^{37-42}$. For generality, each 'site' in the Frenkel-Holstein model represents a moiety (e.g., a phenylene ring or ethynylene bond).

Suppose that the operator $\hat{a}_{n}^{\dagger}$ creates a Frenkel exciton on the $n$th moiety that couples to the local normal coordinate, $Q_{n}$, on the same moiety. If excitations of a normal mode are created by the operator $\hat{b}_{n}^{\dagger}$, then the Frenkel-Holstein model reads,

$$
\begin{aligned}
H_{\mathrm{FH}}= & \sum_{n=1}^{N} \epsilon_{n} \hat{a}_{n}^{\dagger} \hat{a}_{n}+\sum_{n=1}^{N} J_{n}\left(\hat{a}_{n+1}^{\dagger} \hat{a}_{n}+\hat{a}_{n}^{\dagger} \hat{a}_{n+1}\right) \\
& -\frac{1}{\sqrt{2}} \sum_{n=1}^{N} A_{n} \hbar \omega_{n}\left(\hat{b}_{n}^{\dagger}+\hat{b}_{n}\right) \hat{a}_{n}^{\dagger} \hat{a}_{n}+\sum_{n=1}^{N} \hbar \omega_{n} \hat{b}_{n}^{\dagger} \hat{b}_{n},
\end{aligned}
$$

where $N$ is the number of moieties in the macrocycle. The excitation energy of the Frenkel exciton onto the $n$th moiety is $\epsilon_{n}$ and $J_{n}$ is the exciton transfer integral between neighboring moieties. For realistic polymer systems both of these parameters are subject to random fluctuations. $\omega_{n}$ is the angular frequency of the normal mode and $A_{n}$ is the dimensionless exciton-phonon coupling constant.

In this work we fix the on-site energy for each moiety and introduce disorder via modulating the transfer integral, $J_{n}$, as

$$
J_{n}=J_{D D}+J_{S E} \cos ^{2} \phi_{n}
$$

where $J_{D D}$ quantifies the dipole-dipole (through space) and $J_{S E}$ the superexchange (through bond) transfer (for explicit formulations see Appendix A of ef $^{43}$ ). The superexchange term is dependent on the torsional angle between neighboring moieties, $\phi_{n}$, which is chosen to be a Gaussian random variable with standard deviation, $\sigma_{\phi}$. For freely rotating monomers ${ }^{24}$

$$
\sigma_{\phi}=\left(\frac{k_{B} T}{K}\right)^{\frac{1}{2}},
$$

where $K$ is the (harmonic) force constant of the torsional motion of the phenylene ring around the ethynylene bond. The Frenkel-Holstein model parameters for poly(para-phenylene ethynylene) macrocycles are listed in Table I.

For the Frenkel-Holstein model the transition dipole moment operator is defined as

$$
\underline{\hat{\mu}}=\sum_{n=1}^{N} \underline{\mu}_{n}\left(\hat{a}_{n}^{\dagger}+\hat{a}_{n}\right),
$$


where $\underline{\mu}_{n}$, the transition dipole moment of the $n$th moiety, is

$$
\underline{\mu}_{n}=\mu_{0}\left(\cos \left(\frac{2 \pi n}{N}\right), \sin \left(\frac{2 \pi n}{N}\right), 0\right)
$$

for a ring lying in the $x-y$ plane and $\mu_{0}$ is the moiety transition dipole moment.

\section{B. The DMRG Method}

The fully quantized Frenkel-Holstein Hamiltonian spans a Hilbert space too large for direct diagonalization. In order to make the computation feasible we employ the Density Matrix Renormalization Group (DMRG) approach, which is a very accurate and variational truncation method which converges for a finite and reasonably small basis for onedimensional systems, as the ones considered here ${ }^{44-46}$. In this section we briefly summarize how DMRG is used to calculate transition dipole moments; see Section IV A of ref ${ }^{43}$ for details of the application of the DMRG method to the quantum Frenkel-Holstein Hamiltonian.

In the following discussion we denote the eigenstates of the quantum Frenkel-Holstein model, Eq. (1), as $|\mathrm{EX}, \alpha\rangle$, where $\alpha \geq 1$. The lowest eigenstate (i.e., the groundstate) of the quantum Frenkel-Holstein model corresponds to $|\mathrm{EX}, \alpha=1\rangle$, i.e., to the lowest excited exciton in its ground vibrational manifold. Excited states of the quantum Frenkel-Holstein model correspond to $|\mathrm{EX}, \alpha\rangle$ with $\alpha>1$. If there are large electronic gaps (i.e., if $J \gg \hbar \omega$ and $N$ is suitably small) - namely the adiabatic regime - then $2 \leq \alpha \leq N+1$ corresponds to the set of one-phonon vibrational states associated with the lowest excited vibronic manifold. Conversely, if there are small electronic gaps - namely the anti-adiabatic regime - any $|\mathrm{EX}, \alpha \geq 2\rangle$ might correspond to a vibrational ground state of a higher vibronic manifold (see also Section VIB).

The transition dipole moment of a macrocycle of $N$ monomers is

$$
\underline{\mu}=\sum_{n=1}^{N} \underline{\mu}_{n}\left\langle\mathrm{EX}, \alpha\left|\hat{a}_{n}^{\dagger}\right| \mathrm{GS}\right\rangle,
$$

where in general $|\mathrm{GS}\rangle$ belongs to groundstate manifold (and is thus not described by the Frenkel-Holstein model) and $\underline{\mu}_{n}$ is given by Eq. (5). We calculate the absorption transition density from the true electronic and vibrational groundstate, $|\mathrm{GS}, v=0\rangle$, to an arbitrary eigenstate of Eq. (1) via the evaluation of $\left\langle\mathrm{EX}, \alpha\left|\hat{a}_{n}^{\dagger}\right| \mathrm{GS}, v=0\right\rangle$, where $\alpha=1$ corresponds to the $0-0$ transition. 
We calculate the emission transition density from the excited state vibrational ground state, $|\mathrm{EX}, \alpha=1\rangle$, to the electronic groundstate vibrational manifolds via the evaluation of $\left\langle\mathrm{EX}, \alpha=1\left|\hat{a}_{n}^{\dagger}\right| \mathrm{GS}, v_{m}\right\rangle$, where $v_{m}$ means $v$ vibrational excitations on monomer $m$. The $0-1$ emission transition dipole moment is then calculated assuming a localized phonon basis in the groundstate (as described in Section III B of $\operatorname{ref}^{43}$ ).

\section{The Born-Oppenheimer Regime}

In the Born-Oppenheimer regime, where the normal modes are treated as classical variables, the Born-Oppenheimer Hamiltonian equivalent of Eq. (1) is

$$
H_{\mathrm{FH}}^{\mathrm{BO}}=\sum_{n=1}^{N} \epsilon_{n} \hat{a}_{n}^{\dagger} \hat{a}_{n}+\sum_{n=1}^{N} J_{n}\left(\hat{a}_{n+1}^{\dagger} \hat{a}_{n}+\hat{a}_{n}^{\dagger} \hat{a}_{n+1}\right)-\sum_{n=1}^{N} A_{n} \hbar \omega_{n} Q_{n} \hat{a}_{n}^{\dagger} \hat{a}_{n}+\frac{1}{2} \sum_{n=1}^{N} \hbar \omega_{n} Q_{n}^{2},
$$

where $Q_{n}=\left\langle\hat{b}_{n}^{\dagger}+\hat{b}_{n}\right\rangle / \sqrt{2}$ is the dimensionless displacement of the $n$th oscillator. Since $\hbar \omega / J \sim 0.1$ in conjugated polymers, the Born-Oppenheimer regime is expected to be applicable, provided that the excited state energy gaps are not too small, which in practice means sufficiently small macrocycles or large disorder.

The solutions of the Frenkel-Holstein Hamiltonian in the Born-Oppenheimer regime have been derived and discussed fully elsewhere ${ }^{33,43}$ and so we just give a brief summary here. The eigenfunctions of the Frenkel Hamiltonian (i.e., the first two terms on the right-handside of Eq. (7)) are the excitonic wavefunctions, $\Psi_{j}$, with vertical transition energies, $E_{j}^{\text {vert }}$. To obtain the transition energy for the 0-0 transition of the $j$ th state, $E_{j, 00}$, we require the relaxation energy associated with the nuclear relaxation of the molecule, which is given by

$$
E_{j}^{\mathrm{relax}}=\sum_{n=1}^{N} \hbar \omega_{n} S_{n}(1)\left|\Psi_{j}(n)\right|^{4}
$$

where $S_{n}(1)$ is the Huang-Rhys parameter for a single moiety, given as $S_{n}(1)=A_{n}^{2} / 2$. If $\hbar \omega_{n}$ is site-independent we can simplify this expression to

$$
E_{j}^{\mathrm{relax}}=\hbar \omega S_{j}(N),
$$

where

$$
S_{j}(N)=\sum_{n=1}^{N} S_{n}(1)\left|\Psi_{j}(n)\right|^{4}
$$


is an effective Huang-Rhys parameter. For homomoiety systems,

$$
S_{j}(N)=S(1) / \mathrm{IPR}_{j}
$$

where $\mathrm{IPR}_{j}$, the inverse participation ratio, defined by

$$
\mathrm{IPR}_{j}=\frac{1}{\sum_{n=1}^{N}\left|\Psi_{j}(n)\right|^{4}}
$$

is a measure of the spread of the exciton center-of-mass wavefunction.

The transition energy is then

$$
E_{j, 00}=E_{j}^{\mathrm{vert}}-E_{j}^{\mathrm{relax}}
$$

From this we obtain the absorption and emission spectra containing the vibronic progression as

$$
A(E) \propto \sum_{j} \sum_{v} E_{j, 0 v}^{\mathrm{A}}\left|\mu_{j, 0 v}\right|^{2} \delta\left(E-E_{j, 0 v}^{\mathrm{A}}\right)
$$

with

$$
E_{j, 0 v}^{\mathrm{A}}=E_{j, 00}+v \hbar \omega
$$

in the case of absorption and

$$
I(E) \propto \sum_{j} \sum_{v} p_{j, v}\left(E_{j, 0 v}^{\mathrm{E}}\right)^{3}\left|\mu_{j, 0 v}\right|^{2} \delta\left(E-E_{j, 0 v}^{\mathrm{E}}\right)
$$

with

$$
E_{j, 0 v}^{\mathrm{E}}=E_{j, 00}-v \hbar \omega
$$

for emission, where $p_{j, v}$ is the Boltzmann distribution for the state $(j, v) . \delta$ is a line shape function, which is chosen to be a Lorentzian in this work.

In the Born-Oppenheimer-Condon approximation, i.e., assuming both a factorization of the electronic and nuclear wavefunctions and that the electronic transition dipole moment is independent of the normal coordinates, the transition dipole moments, $\mu_{j, 0 v}$, are given $\operatorname{as}^{33,43}$,

$$
\left|\mu_{j, 0 v}\right|^{2}=\left|\underline{\mu}_{j}(N)\right|^{2} F_{j, 0 v},
$$

with

$$
F_{j, 0 v}=S_{j}(N)^{v} \frac{\exp \left(-S_{j}(N)\right)}{v !}
$$


being the effective Frank-Condon factor. The electronic transition dipole moment is

$$
\underline{\mu}_{j}(N)=\sum_{n=1}^{N} \underline{\mu}_{n} \Psi_{j}(n),
$$

where $\underline{\mu}_{n}$ is given by Eq. (5).

We conclude this section by noting that in the Condon approximation,

$$
\frac{I_{j, 00}}{I_{j, 01}}=\frac{A_{j, 00}}{A_{j, 01}}=\frac{F_{j, 00}}{F_{j, 01}}=\frac{1}{S_{j}(N)}=\frac{\mathrm{IPR}_{j}}{\bar{S}(1)},
$$

where, neglecting the small differences in the transition energies between $0-0$ and $0-1$ transitions $I_{j, 0 v} \propto A_{j, 0 v} \propto\left|\mu_{j, 0 v}\right|^{2}$, and $\bar{S}(1)$ is the weighted Huang-Rhys parameter for a monomer. The significance of Eq. (21) is that it shows within the Condon approximation that the absorption and emission intensity ratios are the same and are independent of geometrical factors. This prediction clearly fails for perfectly circular macrocycles, because $A_{j, 00}$ and $I_{j, 00}$ vanish for the lowest exciton state (i.e., $j=0$ ), whereas $I_{j, 01}$ and $A_{j, 01}$ do not. As described in Section III B and Section V, to correctly describe optical transitions in macrocycles it is necessary to include the Herzberg-Teller effect. ${ }^{47}$

\section{ORDERED, CIRCULAR MACROCYCLES: THE HERZBERG-TELLER EF- FECT AND THE ROLE OF TEMPERATURE}

Rotational angular momentum is conserved on a uniform circular ring and hence the

angular momentum quantum number, $j$, labels the states, where $-N / 2<j \leq N / 2$. We denote the ground electronic state as $|\mathrm{GS} ; j\rangle$, where here the angular momentum is carried by the phonons. Similarly, we denote the excited electronic states as $\left|\mathrm{EX} ; j^{\prime}\right\rangle$, where now the angular momentum may be carried by both the exciton and phonons. The transition dipole moment between the ground electronic state and an excited electronic state is

$$
\underline{\mu}=\left\langle\mathrm{EX} ; j^{\prime}|\underline{\hat{\mu}}| \mathrm{GS} ; j\right\rangle \text {. }
$$

From symmetry, $\underline{\mu} \neq 0$ provided that $\left|j-j^{\prime}\right|=1$.

To simplify the discussion, in this section we consider homomoiety macrocycles. Thus, assuming for the moment only excitonic contributions to $|\mathrm{EX} ; j\rangle$, we may write

$$
|\mathrm{EX} ; j\rangle=\sum_{n} \Psi_{j}(n)|n\rangle
$$


where

$$
\Psi_{j}(n)=\frac{1}{\sqrt{N}} \exp (i 2 \pi n j / N)
$$

is the Frenkel exciton center-of-mass wavefunction with energy

$$
E_{j}=\epsilon+2 J \cos \left(\frac{2 \pi j}{N}\right)
$$

and

$$
|n\rangle=\hat{a}_{n}^{\dagger}|0\rangle
$$

is the ket representing a Frenkel exciton on moiety $n$.

Evaluating Eq. (20) for the linear combinations $\Psi_{ \pm}=\left(\Psi_{j=+1} \pm \Psi_{j=-1}\right) / \sqrt{2}$ gives $\mu=$ $\mu_{0} \sqrt{N / 2}$ in orthogonal directions (and hence the total oscillator strength is $\propto N$ ).

This simple analysis shows that - ignoring phonons - absorption occurs into the $\Psi_{j= \pm 1}$ electronic states. In addition, assuming Kasha's rule and relaxation to the $\Psi_{j=0}$ electronic state, emission is dipole forbidden. This section describes how this simple analysis breaks down first, by considering thermally induced emission from the $\Psi_{j= \pm 1}$ states (in Section III A) and second, by considering the Herzberg-Teller effect (in Section III B).

\section{A. The Role of Temperature}

For large macrocycles, i.e., large $N$, the energy gap between the $j=0$ and $j=1$ levels is $4 \pi^{2} J / N^{2}$. Thus, defining the reduced temperature as $T_{\text {red }}=4 \pi^{2} J / k_{B} N^{2}$, we show in Fig. 2 the relative thermal population of the $j=1$ level versus $T / T_{\text {red }}$. Evidently, $p_{j=1}$ reaches

a maximum at $T \sim T_{\text {red }}$ before decreasing at larger $T / T_{\text {red }}$ as higher energy levels become more populated. Indeed, since the spectrum is bounded, for $T \gg T_{\text {red }}, p_{j=1} \rightarrow 1 / N$.

Since, in the Condon approximation, only the $j=1$ level emits, the emission intensity is $N \times p_{j=1}$ and this is also shown in Fig. 2. Evidently, as either $T$ increases or $T_{\text {red }}$ decreases (i.e., $N$ increases) the intensity becomes independent of $T$ or $N$.

\section{B. The Herzberg-Teller Effect}

The prediction that the totally-symmetric $(j=0)$ electronic state is dipole forbidden arises from the assumption that the electronic transition dipole moment is independent of the normal coordinates. This is the Condon approximation. The Herzberg-Teller ${ }^{48}$ correction 


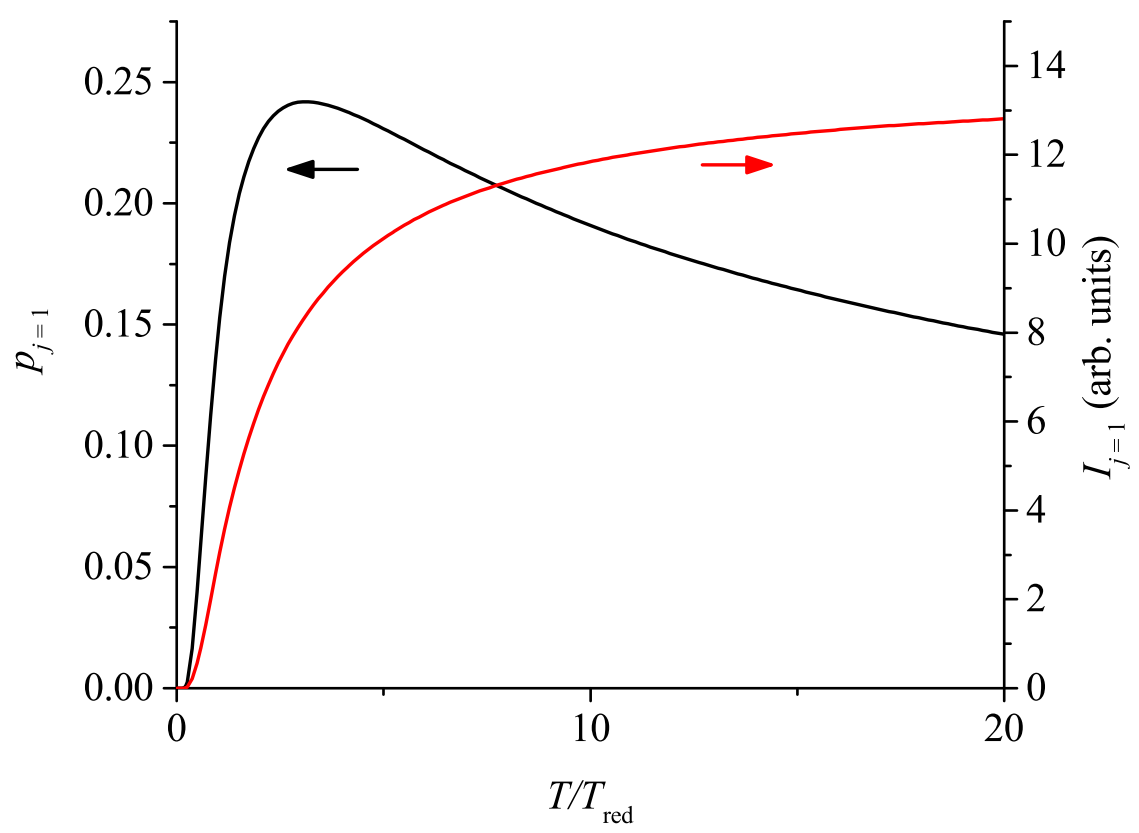

FIG. 2: Relative thermal population (black, left) and emission intensity (red, right) of the $j=1$ level of an ordered macrocycle against $T / T_{\text {red }}$, where the reduced temperature is $T_{\text {red }}=$ $4 \pi^{2} J / k_{B} N^{2}$.

to the Condon approximation is to perform a first order Taylor expansion of the electronic transition dipole moment in the normal coordinates. As a consequence, the 0-1 transition associated with the $j=0$ electronic state becomes weakly allowed due to intensity borrowing from the $j= \pm 1$ states. As shown in Appendix A, treating the Herzberg-Teller correction perturbatively, we find that the $I_{01}$ emission intensity from the vibrational ground level of the $j=0$ electronic state to the first vibrational excited level of the electronic ground state is given by

$$
I_{01}^{H T} \propto N^{4} \mu_{0}^{2} A^{2}\left(\frac{\hbar \omega}{J}\right)^{2} .
$$

This result is expected to be valid provided that the perturbation theory is valid ${ }^{49}$, i.e.,

$$
\left(\frac{\hbar \omega}{J}\right) \frac{A}{N^{3 / 2}} \ll 1 .
$$

We test the validity of Eq. (27) by solving the fully quantized Frenkel-Holstein model for homomoeity macrocycles employing the DMRG routine. We note that this is an essentially exact calculation, which goes beyond the Born-Oppenheimer approximation. The results 

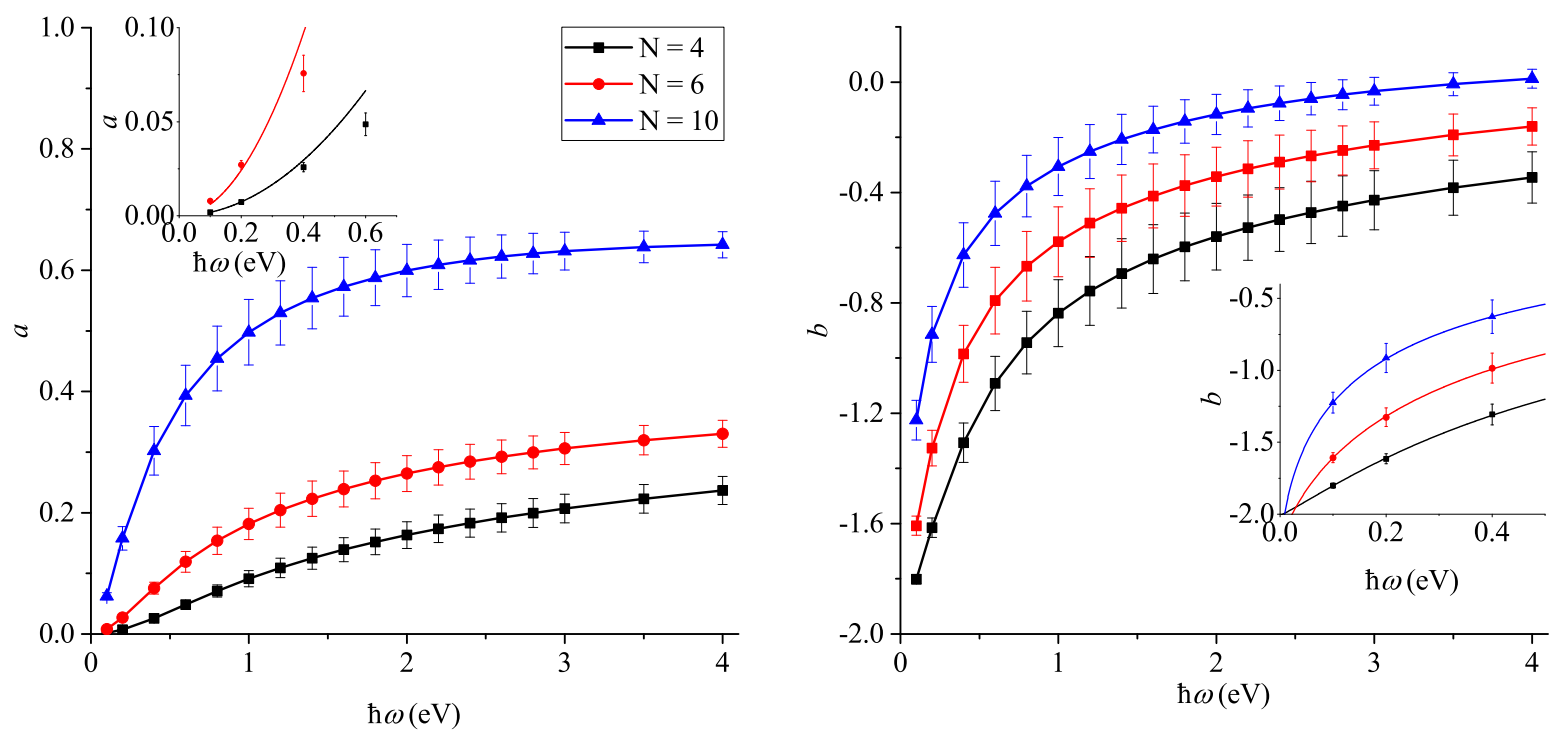

FIG. 3: Evolution of the fitting parameters of Eq. (29): $a$ (left) and $b$ (right) with $\hbar \omega$ and ring size, obtained from DMRG solutions of the quantized Frenkel-Holstein model, Eq. (1). The insets show the $\hbar \omega \rightarrow 0$ regime with extrapolation. $a$ is quadratic in $\hbar \omega$ and $b \rightarrow 2$ for the adiabatic regime. The curves in the graph are a guide to the eye, the graphs in the inset are quadratic and logistic fits, respectively.

were fitted for varying $N$ and $\hbar \omega$ as

$$
I_{01}^{H T}=a J^{b}
$$

with the fitting parameters $a$ and $b$, which are plotted in Fig. 3. As can be seen, in the adiabatic limit $(\hbar \omega / J \rightarrow 0)$ the parameters $a$ and $b$ tend to 0 and -2 , respectively. To a good approximation, $a$ is quadratic in the adiabatic regime and therefore $a \propto(\hbar \omega)^{2}$. Hence,

$$
I_{01} \propto\left(\frac{\hbar \omega}{J}\right)^{2} ; \quad \hbar \omega / J \ll 1,
$$

which confirms our result, Eq. (27). We also see that in the adiabatic regime the prediction of Eq. (27) fails more quickly as $\hbar \omega$ is increased for larger $N$

\section{Comments}

- Although not the main topic of this paper, the results presented in Fig. 3 indicate that in the anti-adiabatic regime (i.e., $\hbar \omega / J>1$ or large $N$ ) the Herzberg-Teller correction to the $0-1$ emission intensity $I_{01}^{\mathrm{HT}} \propto N$ and independent of $\hbar \omega / J$. 
- As shown in the next three Sections, broken symmetry renders the lowest excited state dipole active within the Condon approximation and hence $I_{01}$ is enhanced by the Condon term. However, as shown in Section V, even with broken symmetry the Herzberg-Teller correction remains a significant contribution to the $0-1$ emission intensity.

- The Herzberg-Teller correction for absorption in a uniform CPPE ring is discussed in Section VIB.

\section{THE ROLE OF BROKEN SYMMETRY}

In the last section, the role of temperature in a perfectly ordered macrocycle was to simply thermally populate the $j=1$ level, leading to thermally enhanced emission (as shown in Fig. 2). More generally, however, for macrocycles with freely rotating monomers thermal energy will cause torsional disorder given by Eq. (3), leading to a breakdown of the symmetry-determined selection rules.

In this section we investigate the role of static torsional disorder on the optical properties of macrocycles. We take the disorder, $\sigma_{\phi}$, as a model parameter in the exciton transfer integral, Eq. (2), of the Frenkel-Holstein model and we use CPPE macrocycles as our model system. The model parameters for CPPE are listed in Table I. As we are concerned with the role of broken symmetry, we solve the Frenkel-Holstein model in the Condon approximation, as described in Section II C. (The combined roles of broken symmetry and Herzberg-Teller corrections are described in the next section.)

Before describing the optical properties, we first review the role of disorder in localizing states in one-dimensional systems. As is well-established, the role of disorder in onedimensional systems is highly non-perturbative: any amount of disorder exponentially localizes states ${ }^{30,31,50}$. Rather remarkably, in one-dimensional systems there are a class of states in the low energy tail of the density of states that are superlocalized, named local exciton ground states (LEGSs) ${ }^{25,51}$. Local exciton ground states are essentially nodeless and non-overlapping wavefunctions that together spatially span the entire ring. They define chromophores in linear and cyclic polymers ${ }^{55}$. In a linear polymer (neglecting curvature effects), the oscillator strength is proportional to the chromophore length. As shown in Appendix B, in macrocycles, however, the oscillator strength is proportional to the square 


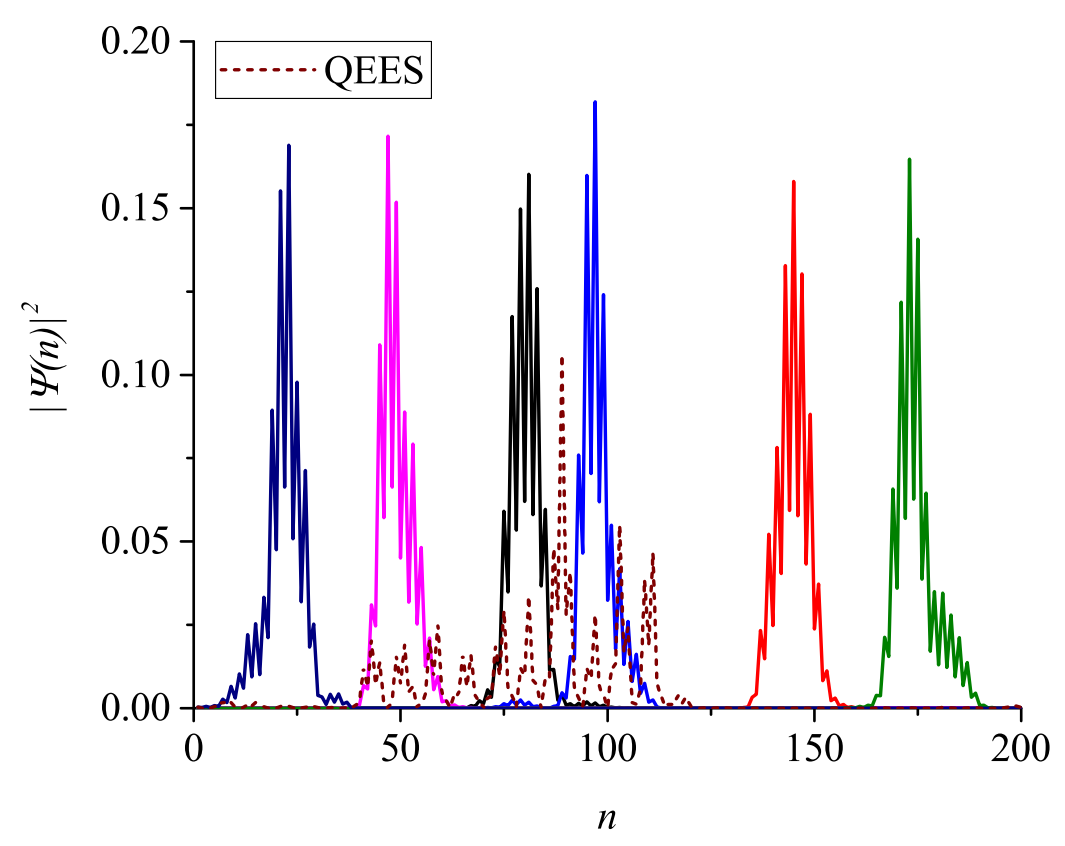

FIG. 4: Excitonic density showing six LEGSs (solid lines) and one QEES (dashed line) on a CPPE ring consisting of 200 monomers and torsional disorder, $\sigma_{\phi}=0.3$ radians. LEGSs are quasi non-overlapping and spacefilling, and define chromophores, whereas QEESs span multiple LEGSs.

of the chord length, $L$, joining the ends of the chromophore, as illustrated in Fig. 7. Higher lying states are also localized, but will be nodeful and generally spatially overlap a number of low-lying LEGSs. These states are named quasiextended exciton states (QEESs). On a macrocycle the ultra-fast interconversion of QEESs to LEGSs is manifest as ultra-fast fluorescence depolarization ${ }^{42,53}$. Figure 4 illustrates the excitonic density in LEGSs and a QEES on a 200 monomer CPPE macrocycle. For this choice of $\sigma_{\phi}$ there are six LEGSs spanning the circumference of the ring.

The spatial spread of a LEGS is quantified by the inverse participation ratio, IPR, defined by Eq. (12). Figure 5 shows the average value of IPR for LEGSs as a function of disorder for two cyclo(para-phenylene ethynylene) (CPPE) macrocycles of different size. [15]CPPE consists of 15 monomers (i.e., 15 phenylene and 15 ethynylene units) and [30]CPPE is twice that. For $\sigma_{\phi} \gtrsim 0.2$ radians, the size of the macrocycle no longer determines the size of the LEGSs.

We now turn to consider the optical properties. Figure 6 shows the evolution of the 


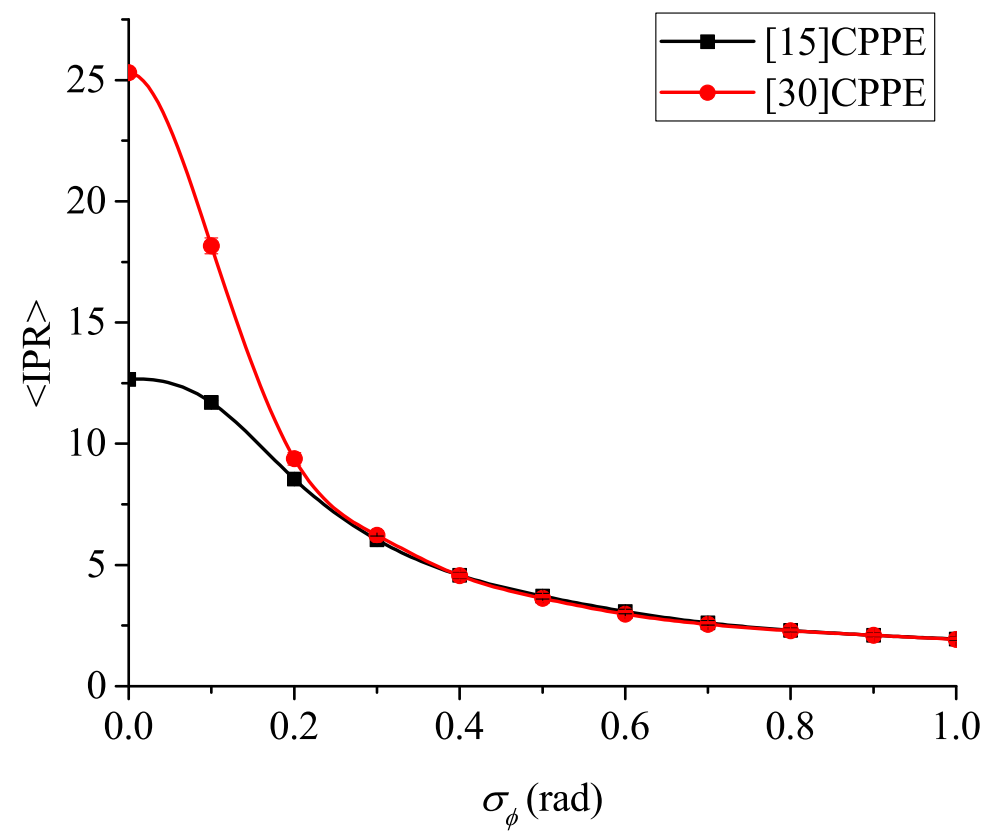

FIG. 5: The spatial average spread of LEGSs (in monomer units), defined by $\langle$ IPR $\rangle$, with increasing disorder for [15]CPPE and [30]CPPE. (Note that for heteromoiety macrocycles IPR $\neq N$ for ordered rings.)

squared transition electronic dipole moment of the lowest two excited states of [15]CPPE and [30]CPPE as a function of disorder. The decrease of the transition dipole moment in the $j=$ 1 state with increasing disorder is simultaneously accompanied by acquisition of oscillator strength in the $j=0$ state. As can be clearly seen for the larger macrocycle, the $j=0$ state does not monotonically acquire oscillator strength. This can be understood by recalling that for a nodeless exciton wavefunction the oscillator strength is (approximately) linearly proportional to the square of the length of the chord joining the ends of the chromophore (as shown in Fig. 7). Thus, for small disorder the intensity increases with disorder, $\sigma_{\phi}$, because the chromophore is decreasing in size and the chord length is increasing (i.e., $\theta$, as defined in Fig. 7, satisfies $\pi \leq \theta<2 \pi$ ). However, once $\theta<\pi$, for larger disorder the chord length decreases with increasing disorder, and hence the intensity begins to decrease. For [30]CPPA this occurs when $\sigma_{\phi} \simeq 0.35$ radians. Figure 6 also shows that for large disorder the intensities of the $j=0$ and $j=1$ states are essentially the same and independent of $N$, because they are both LEGSs that only partially span the circumference. 


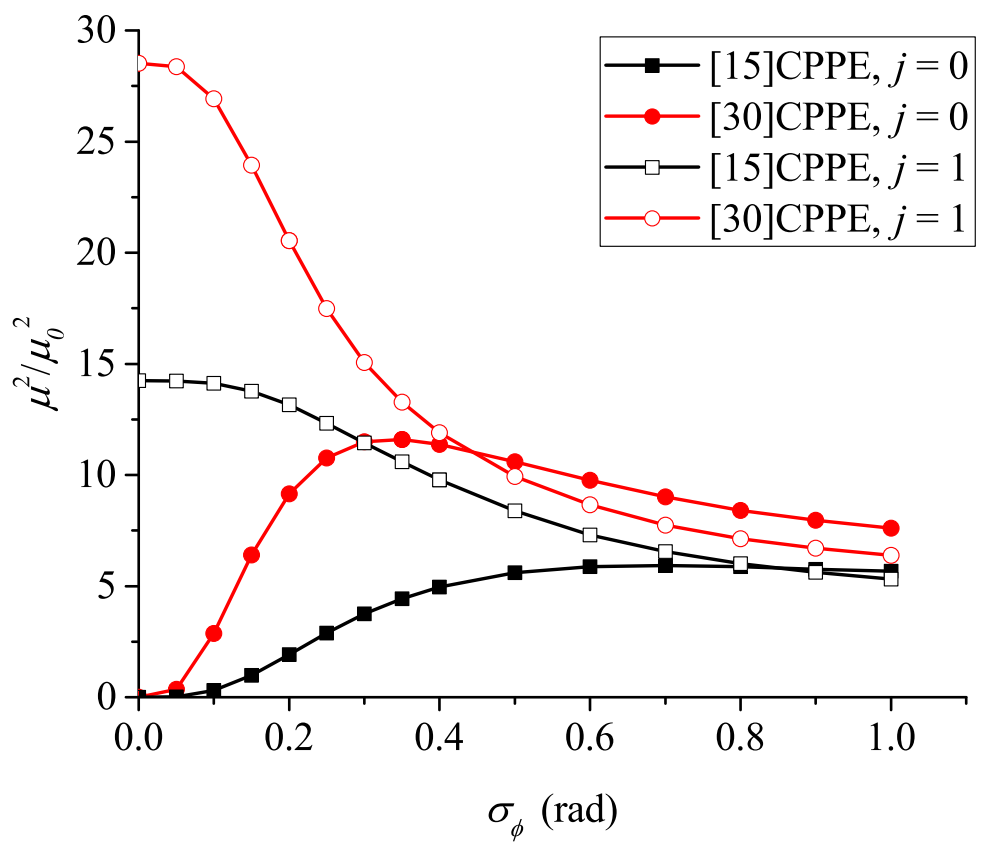

FIG. 6: The squared electronic transition dipole moment of the $j=0$ and $j=1$ states for [15]CPPE and [30]CPPE as a function of disorder. Clearly visible is the acquisition of oscillator strength in the $j=0$ state until the average conjugation length becomes less than half of the ring circumference. (Except for the case $\sigma_{\phi}=0, j$ is not an angular momentum quantum number.)

\section{THE ROLES OF BROKEN SYMMETRY AND CURVATURE}

In this section we draw together two of the themes of Sections III and IV, namely the role of broken circular symmetry in rendering the lowest excited state dipole allowed within the Condon approximation and the role of the Herzberg-Teller effect in increasing the emissive intensity of the $0-1$ transition relative to the $0-0$ transition from this state.

To quantify the discussion we illustrate in Fig. 7 a LEGS localized on a section of a macrocycle of radius $R$ and circumference $N=2 \pi R$. We identify the angle corresponding to the spread of the LEGS as the ratio of the conjugation length (in units of the monomer length) normalized to the circumference of the ring, i.e.,

$$
\theta=2 \pi \frac{\langle\mathrm{CL}\rangle}{N}
$$

$\langle C L\rangle / N \rightarrow 0$ corresponds to an increasing linearization, whereas $\langle C L\rangle / N=1$ corresponds to a fully circumventing LEGS. 


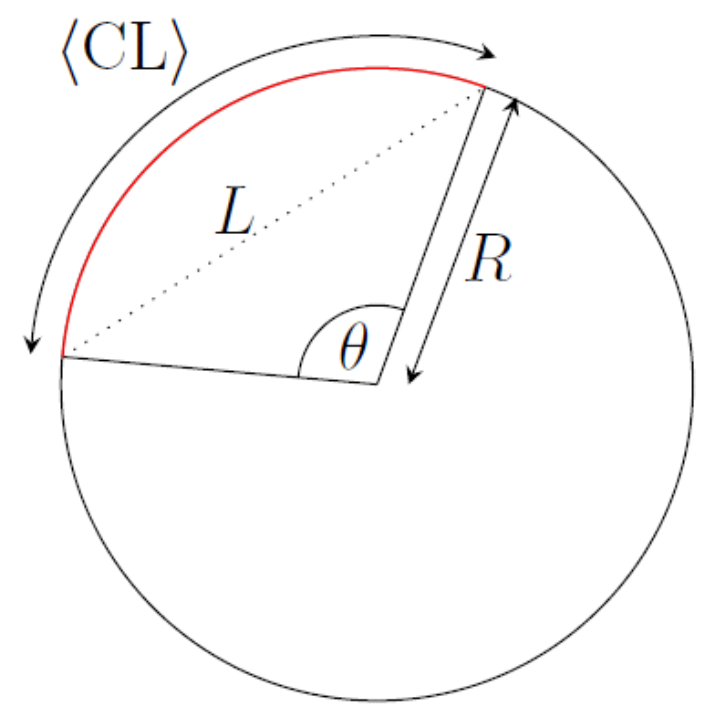

FIG. 7: Schematic representation of LEGS with a conjugation length, $C L$, (red segment) on a macrocycle of radius $R$. The LEGS spans a chord (dashed line) of length $L$, which determines $I_{00}$.

As discussed in Section II C, in the Condon approximation the $I_{00} / I_{01}$ emission intensity ratio is

$$
\frac{\left\langle I_{00}\right\rangle}{\left\langle I_{01}\right\rangle}=\frac{\langle\mathrm{IPR}\rangle}{S(1)},
$$

where IPR is defined in Eq. (12). We note that this result applies for the emission from any state and is independent of the geometry of the system. It evidently fails for a uniform macrocycle because, as described in Section III B, for the lowest excited state $I_{00}$ vanishes whereas $I_{01}$ does not. We assume Kasha's law and thus the averages in Eq. (32) are taken over the ensemble of (equally populated) LEGSs.

We now define $X$ as the intensity ratio scaled by the mean inverse participation ratio, i.e.,

$$
X=\frac{\left\langle I_{00}\right\rangle}{\left\langle I_{01}\right\rangle\langle\mathrm{IPR}\rangle} .
$$

Deviations of $X$ from a constant value indicate deviations from the Condon expression, Eq. (32). Fig. 8 shows the computed value of $X$ versus $\langle\mathrm{CL}\rangle / N$ for different ensembles of disorder for [15]CPPE macrocycles. The results were obtained via the DMRG solutions of the fully quantized Frenkel-Holstein model, and thus include corrections beyond the Condon approximation. The results for a fixed $\hbar \omega / J_{0}$, when scaled appropriately, collapse onto universal curves. However, $X$ decreases more quickly as a function of $\theta$ for larger values 


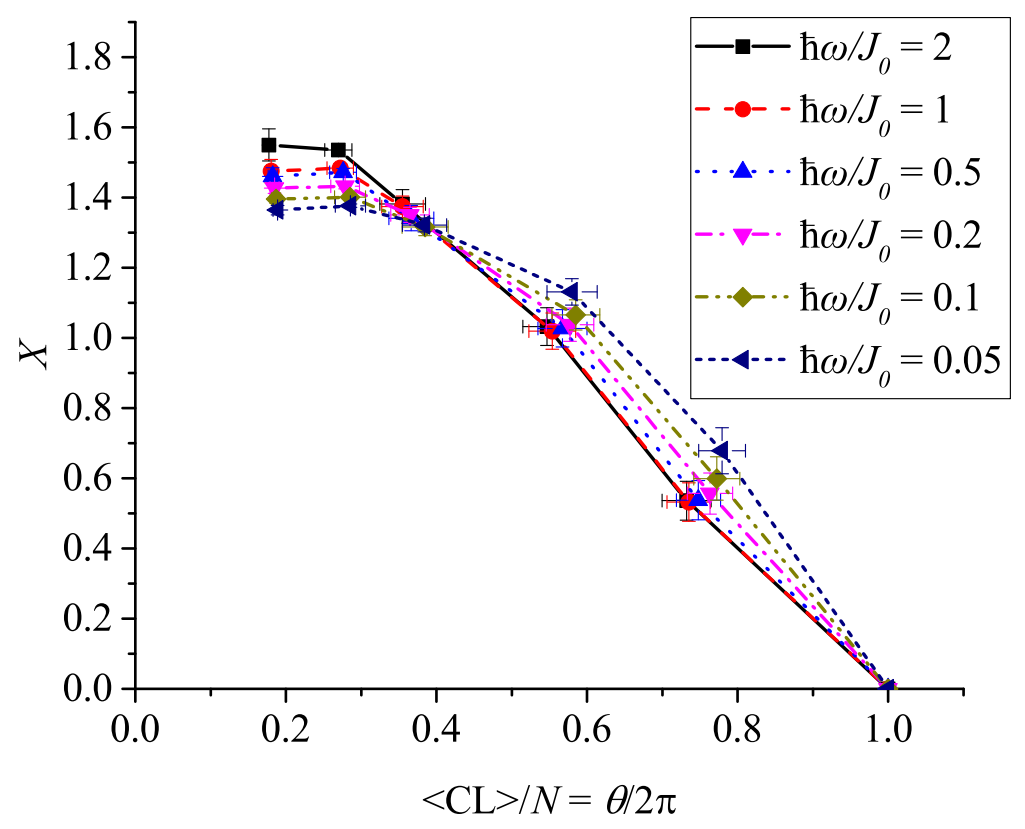

FIG. 8: The ensemble-averaged scaled intensity ratio, $X=\left\langle I_{00}\right\rangle /\left\langle I_{01}\right\rangle\langle\mathrm{IPR}\rangle$, versus increasing curvature of the emitting LEGS (see Fig. 7) for various values of $\hbar \omega / J_{0}$. These results are obtained for [15]CPPE macrocycles via DMRG computations of the quantized Frenkel-Holstein model. The behavior of $X$ is qualitatively predicted by Eq. (C4) and Eq. (C5) and is a consequence of the Herzberg-Teller correction to the Condon approximation (see Appendix C).

of $\hbar \omega / J_{0}$. Setting $J_{0}=J_{D D}+J_{S E} \cos ^{2}(0)=J_{D D}+J_{S E}$ allows us to combine $J$ and $\hbar \omega$ into one factor and ease the discussion, as it quantifies the adiabaticity of the system. For $\hbar \omega / J_{0}<1$ the system is in the adiabatic regime, whereas $\hbar \omega / J_{0}>1$ is the anti-adiabatic regime. Moreover the results presented in Fig. 8 do not depend on any other parameters.

As shown in Appendix C, when the LEGSs almost span the ring, i.e., for $(2 \pi-\theta) \ll 1$,

$$
\frac{\left\langle I_{00}\right\rangle}{\left\langle I_{01}\right\rangle\langle\mathrm{IPR}\rangle}=\frac{1}{\left(S(1)+2(S(1) B)^{1 / 2} \frac{\theta}{(2 \pi-\theta)}+B\left(\frac{\theta}{(2 \pi-\theta)}\right)^{2}\right)}
$$

where $B$ is proportional to the Herzberg-Teller correction, $I_{01}^{\mathrm{HT}}$. The right-hand-side of Eq. (34) evidently vanishes when $\theta=2 \pi$. However, we also see that it vanishes more quickly as a function of $\theta$ for a larger value of the Herzberg-Teller correction term, $B$. As shown in Section III B, this correction is an increasing function of $\hbar \omega / J_{0}$, and thus Eq. (34) qualitatively reproduces Fig. 8. 


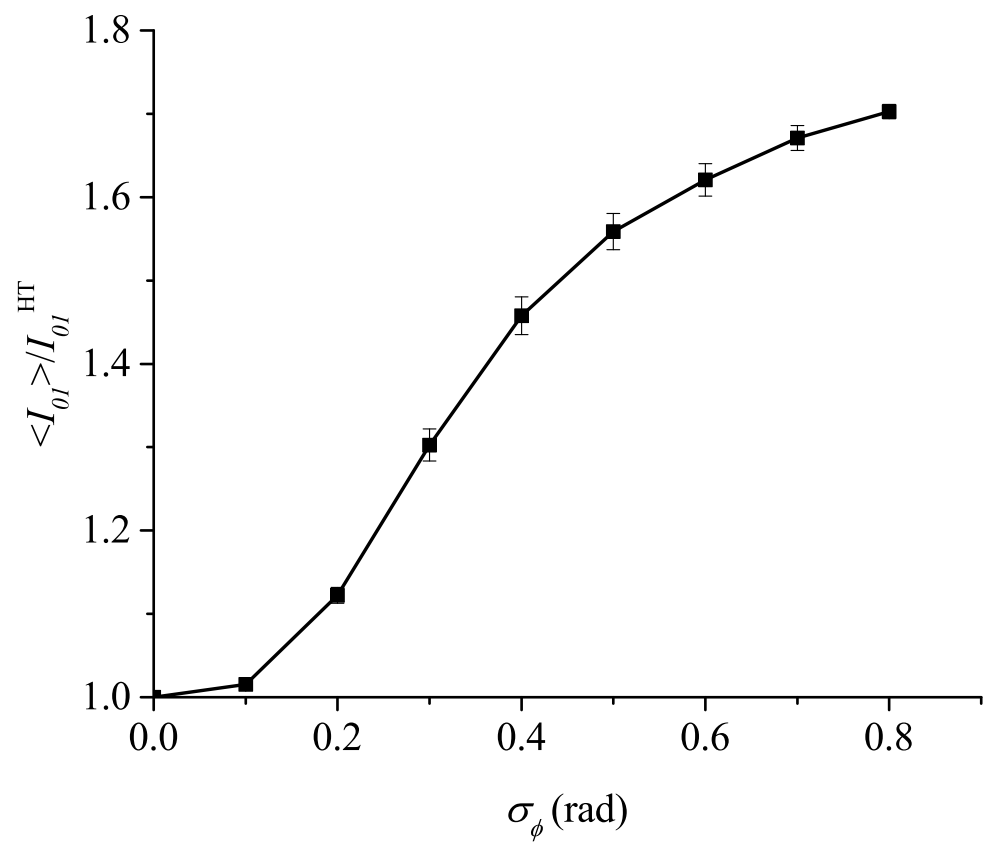

FIG. 9: The ensemble averaged $I_{01}$ normalized by the Herzberg-Teller correction, $I_{01}^{\mathrm{HT}}$, as a function of disorder. These results are obtained for [15]CPPE macrocycles via DMRG computations of the quantized Frenkel-Holstein model. The ensemble average is over LEGSs.

As is also evident from Eq. (C4) and Fig. 8, the scaled intensity ratio, $X$, is a weak function of $\theta$ for small $\theta$, and becomes constant in the adiabatic limit $\left(\hbar \omega / J_{0} \rightarrow 0\right)$. This implies that curvature effects are negligible for large macrocycles with strong disorder and thus (as for linear polymer chains ${ }^{33}$ ) Eq. (32) may be used to determine chromophore sizes.

Our results, shown in Fig. 8 and Eq. (34), are in qualitative agreement with the predictions of Hestand and Spano (shown in Fig. 3 and Eq. (24) of $\operatorname{ref}^{32}$ ), who investigated the effect of curvature on the optical properties of polymers. We do not find quantitative agreement, because our results apply in the adiabatic regime, whereas theirs apply in the anti-adiabatic regime.

The role of broken symmetry and curvature is also illustrated by Fig. 9. This shows the ensemble averaged $I_{01}$ for [15]CPPE normalized by the Herzberg-Teller correction, $I_{01}^{\mathrm{HT}}$, as a function of disorder. As indicated by Eq. (C2), for uniform rings $I_{01} / I_{01}^{\mathrm{HT}}=1$, because $I_{00}$ - and hence $I_{01}^{C}$ - vanish. However, as the disorder is increased and the circular symmetry is broken $I_{00}$ gains intensity, and thus $I_{01}$ gains further intensity via the Condon term, Eq. (32). 


\begin{tabular}{lr}
\hline Parameter & Value \\
\hline Phenylene on-site energy, $E_{P}$ & $6.05 \mathrm{eV}$ \\
Ethynylene on-site energy, $E_{E}$ & $10.4 \mathrm{eV}$ \\
Superexchange exciton transfer integral, $J^{S E}$ & $1.68 \mathrm{eV}$ \\
Dipole-dipole exciton transfer integral, $J^{D D}$ & $0.6 \mathrm{eV}$ \\
Phonon energy, $\hbar \omega$ & $0.2 \mathrm{eV}$ \\
Phenylene exciton-phonon coupling constant, $A_{P}$ & 1.7 \\
Ethynylene exciton-phonon coupling constant, $A_{E}$ & 2.4 \\
Phenylene Huang-Rhys parameter, $S_{P}(1)$ & 1.45 \\
Ethynylene Huang-Rhys parameter, $S_{E}(1)$ & 2.88 \\
Mean dihedral angle (ground and excited states), $\phi_{0}$ & $0^{\circ}$ \\
Elastic torsional force constant ${ }^{1}, K$ & $0.38 \mathrm{eV}^{-2}$ \\
\hline
\end{tabular}

TABLE I: Parameters used in the Frenkel-Holstein model (Eq. (1)) for the calculation of the CPPE spectra. For details of the parametrization, see $\mathrm{ref}^{33} .{ }^{1}$ The force constant was found using ab-initio calculations at the $(\mathrm{HF} / / 6-311 \mathrm{G})$ level.

For high disorder the system saturates, approaching a limiting value of about $\sim 1.8 I_{01}^{\mathrm{HT}}$. This trend resembles the acquisition of $I_{00}$ intensity for the $j=0$ state shown in Fig. 6. Rather significantly, at least for [15]CPPE macrocycles, we see that the Herzberg-Teller term is the dominant contribution to the $0-1$ emission intensity.

\section{MODELING EXPERIMENTAL SPECTRA}

We now turn to describe our predictions for the absorption and emission spectra of conformationally disordered [15] CPPE macrocycles, interpreting them using the insights of the last three sections. The parameterization of the Frenkel-Holstein model for CPPE macrocycles follows the same procedure as described in the appendix of ref ${ }^{33}$ and the parameters are shown in Table I. We examine both the roles of broken symmetry and the Herzberg-Teller effect. Thus, we first describe our predictions assuming the Condon approximation (in Section VI A), before describing the full Frenkel-Holstein model results (in Section VIB), which includes the Herzberg-Teller effect. 


\section{A. Assuming the Condon Approximation}

The results of this section assume the Condon approximation, as described in Section II C. We also adopt Kasha's rule and assume that emission only occurs from LEGSs. We do not consider the role of temperature, because typically for the size of the systems described here, QEESs lie too high in energy above LEGSs to be thermally populated at room temperature.

Figure 10 shows the calculated absorption spectrum for [15]CPPE for different amounts of disorder. Room temperature corresponds to $\sigma_{\phi} \approx 0.25 \mathrm{rad}$. We observe a vanishing of the spectral features in the high-energy tail for increasing disorder with the vibronic peaks being smeared out. Simultaneously, a shoulder at the low-energy edge becomes more intense. At room temperature the shoulder is at about $3.35 \mathrm{eV}(370 \mathrm{~nm})$ and the intense peak is at $3.5 \mathrm{eV}(354 \mathrm{~nm})$. Experimental spectra ${ }^{54}$ show the same features: the absorption spectrum of [14]CPPE has its maximum at about $350 \mathrm{~nm}(3.54 \mathrm{eV})$ and a shoulder at $390 \mathrm{~nm}(3.18$ $\mathrm{eV})$. The position of the maximum does not vary with increasing ring size from [10]CPPE to [14] CPPE, so it can be assumed that for [15]CPPA the position will not change significantly. As for the shoulder, the wavelength is a weak function of the ring size and we thus expect the shoulder for [15]CPPE to be at about $395 \mathrm{~nm}(3.14 \mathrm{eV})$. We reproduce both the intense peak and the shoulder, with wavelengths of about $360 \mathrm{~nm}(3.45 \mathrm{eV})$ and $385 \mathrm{~nm}(3.22 \mathrm{eV})$ for the maximum and shoulder, respectively, showing a shift of about $10 \mathrm{~nm}$ to shorter wavelengths. The origins of the low-energy shoulder and peak are discussed below.

Figure 11 shows the calculated emission spectra for [15]CPPE at the same values of disorder. In the Condon approximation, the emission spectrum for a perfectly ordered system $\left(\sigma_{\phi}=0.0\right)$ vanishes. We once again see a vanishing of spectral features, as well as an increase in intensity with increasing disorder. The experimental spectra ${ }^{54}$ for shorter rings show two main features: a maximum at about $445 \mathrm{~nm}(2.79 \mathrm{eV})$ and a secondary peak at $410 \mathrm{~nm}(3.02 \mathrm{eV})$ for $[14] \mathrm{CPPE}$. The wavelengths of the peaks decrease with increasing ring size, so for [15]CPPE these peaks are expected to be even further blue-shifted. We find in our calculated spectra a single peak with a shoulder due to the vibronic progression. The

peak is at about $380 \mathrm{~nm}(3.26 \mathrm{eV})$, which is $0.24 \mathrm{eV}$ too high in energy for the secondary peak and $0.47 \mathrm{eV}$ too high for the emission maximum. A small shoulder is located $0.2 \mathrm{eV}$ below our main peak. This energy shift compared to the experimental spectrum can have many reasons, one of which being the neglect of bathochromic solvent effects. Furthermore, 


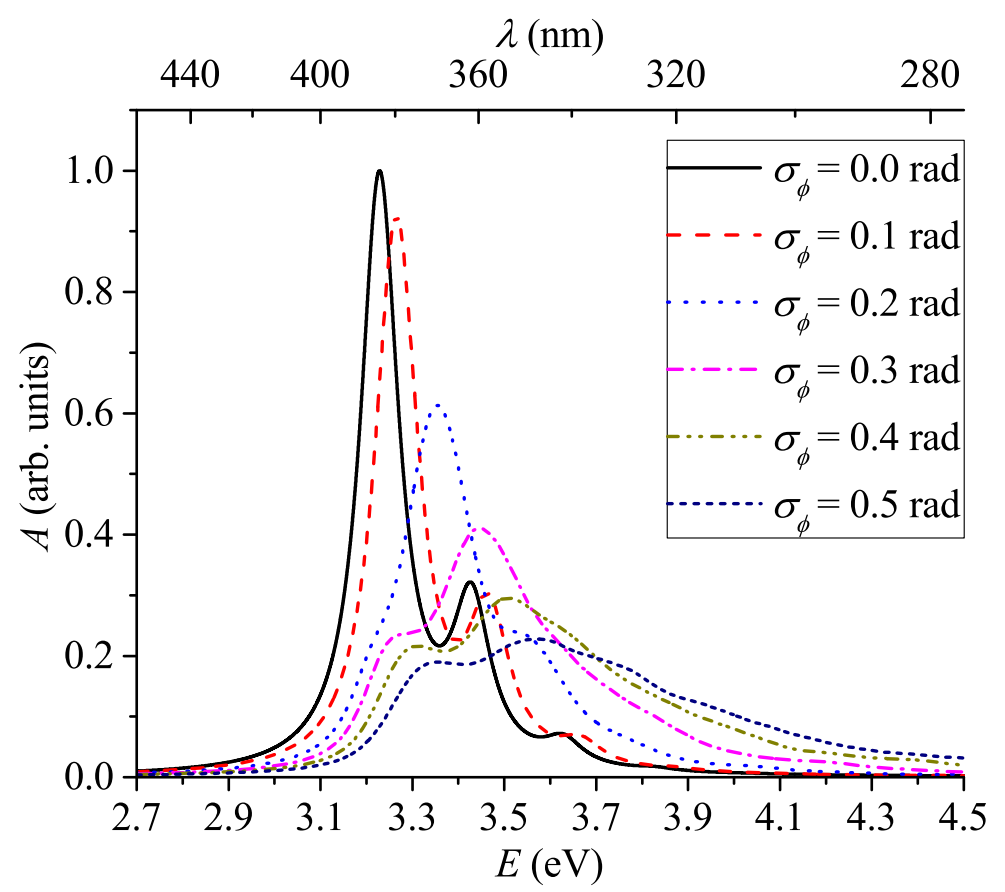

FIG. 10: Absorption spectra for [15]CPPE for different values of disorder, averaged over an ensemble of 1000 rings, calculated within the Condon approximation. (For the ordered ring the absorption spectrum is the vibronic progression of the $j= \pm 1$ states.)

the discrepancy in the intensities (the low-energy peak in the experiment is about 0.8 times the intensity of the high-energy peak) shows that the Condon approximation fails to predict the emission accurately, because of its neglect of the Herzberg-Teller effect.

The peaks seen in the absorption spectrum for the ordered macrocycle $\left(\sigma_{\phi}=0.0 \mathrm{rad}\right)$ correspond to the $j=1$ vibronic progression, with $j=0$ being a dark state. Once excited, the exciton relaxes into the $j=0$ state and as the transition back to the ground state is dipole forbidden, it relaxes non-radiatively, resulting in a vanishing emission spectrum. For disordered systems $\left(\sigma_{\phi}>0.0 \mathrm{rad}\right)$ the $j=0$ state becomes dipole allowed, and will thus contribute to the absorption spectrum and give rise to a non-zero emission spectrum.

Figure 6 shows the transition dipole moment of the $j=0$ and $j=1$ states for both [15]CPPE and [30]CPPE against disorder, indicating that the $j=0$ state gains oscillator strength before saturating while the $j=1$ state loses oscillator strength. We can thus explain the absorption spectra as follows: the initially dark state $j=0$ becomes dipole allowed with disorder and gains oscillator strength and thus appears at the low-energy edge 


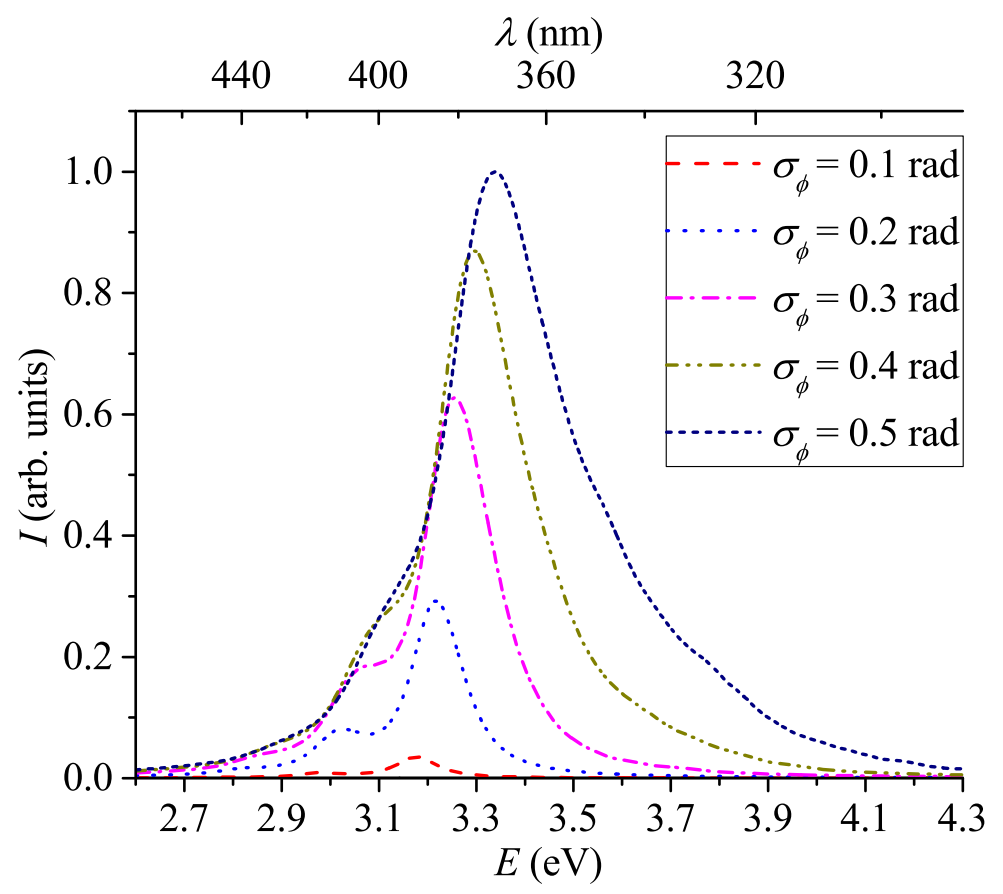

FIG. 11: Emission spectra for [15]CPPE at different disorder, averaged over an ensemble of 1000 rings, calculated within the Condon approximation. (For the ordered ring there is no radiative transition in the Condon approximation.)

of the absorption spectrum. Since the energy gap between the $j=0$ and $j= \pm 1$ states is only $0.09 \mathrm{eV}$ for an ordered ring, these peaks merge as the disorder increases, eventually forming the low-energy 0-0 shoulder for large disorder. Similarly, as the disorder increases and the chromophore sizes decrease, the 0-1 transition gains intensity (as shown by Eq. (21)), and thus the higher energy peak at large disorder is the $0-1$ transition to $j=0, \pm 1$ states. Likewise, an emission spectrum will only be observed for disordered chains as the $j=0$ state becomes dipole allowed and will continue to gain intensity before saturating.

The blue shift of the absorption spectra with increasing disorder is caused by the exciton band narrowing, while the increase in the inhomogenous line width is a consequence of exchange narrowing. Both of these effects are described in detail in Section V.B.3. of ref ${ }^{33}$. 


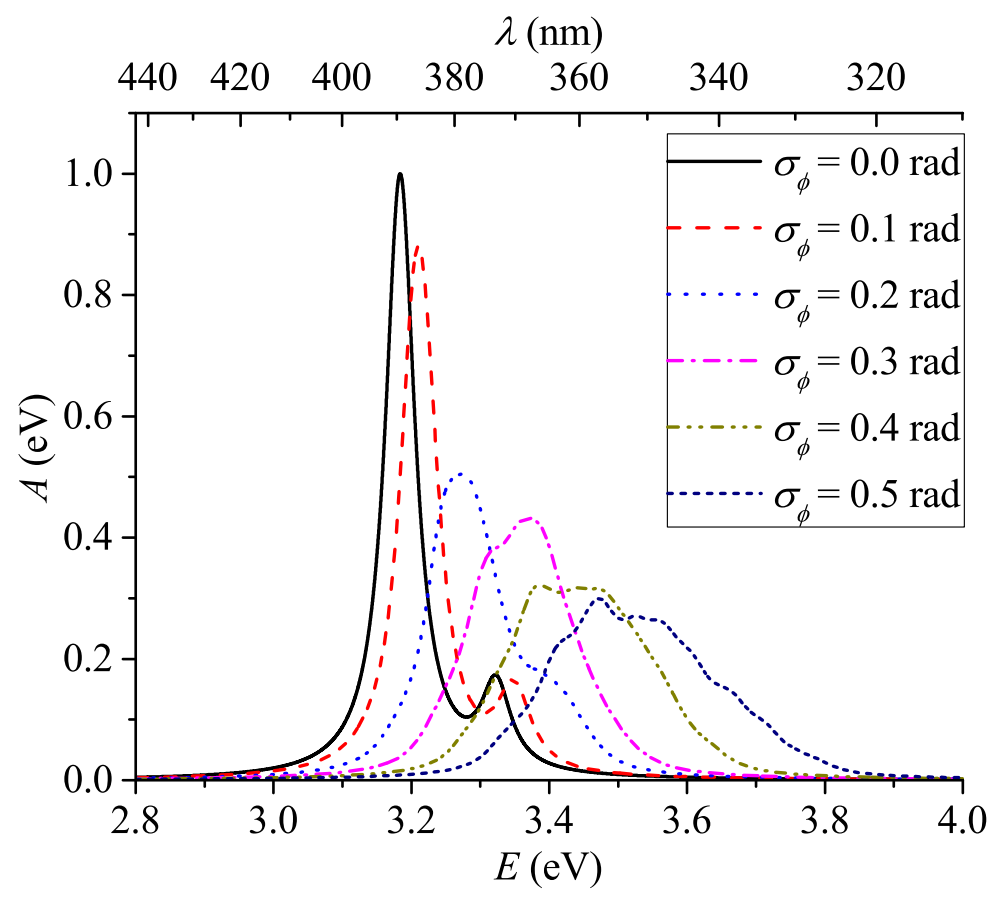

FIG. 12: Absorption spectra of [15]CPPE for different amounts of disorder calculated with the fully quantized Frenkel-Holstein model using a DMRG routine. (For the ordered ring the lowest energy peak is the Condon-allowed transition to the $j= \pm 1, v=0$ states, while the higher energy peak is the Condon-forbidden (i.e., the Herzberg-Teller effect) transition to the $j=0, v=1$ manifold. Higher energy vibronic transitions are not computed.)

\section{B. Beyond the Condon Approximation: The Fully Quantized Model}

The same system was investigated using the fully quantized model solved using the DMRG method, as described in Section II B. In contrast to the results above, owing to the computational costs of the calculations the DMRG results are averaged over 40 different realizations of the disorder. Figures 12 and 13 show the calculated absorption and emission spectra, respectively. In general, 35 states were calculated for [15]CPPE. For an ordered ring the first state corresponds to the $j=0, v=0$ transition; the second and third states to the $j= \pm 1, v=0$ transitions; and the states $4-33$ correspond to the $j=0, v=1$ transitions. (The $j= \pm 1, v=1$ transitions correspond to states $34-93$, which are not practical to calculate.) We note that our DMRG code only computes the 0-0 and 0-1 transitions in emission. 


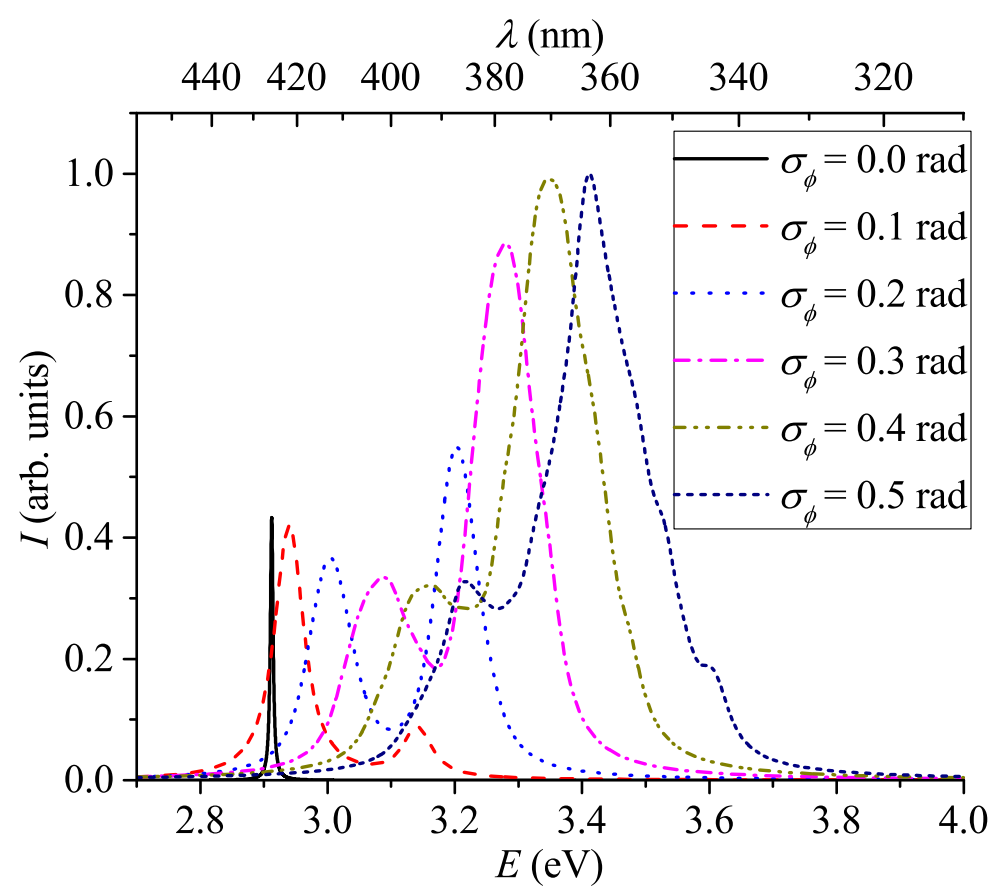

FIG. 13: Emission spectra of [15]CPPE for different amounts of disorder calculated using the fully quantized Frenkel-Holstein model using a DMRG routine. (For the ordered ring the peak at $\sim 2.9$ $\mathrm{eV}$ is the Herzberg-Teller effect, corresponding to emission from the $j=0, v=0$ excited state to the $v=1$ manifold of the ground state.)

The same general trends are reproduced for the absorption spectra as in the Condon approximation: with increasing disorder we see a broadening of the peaks. In contrast to the Condon approximation, however, for the ordered ring we now compute a peak at $\sim 3.3 \mathrm{eV}(376 \mathrm{~nm})$, which is $\sim 0.1 \mathrm{eV}$ above the lowest absorption peak (that arises from transitions to the $j= \pm 1, v=0$ states), and thus $\sim 0.2 \mathrm{eV}$ above the dark $j=0, v=0$ state. This higher energy peak can be assigned to transitions to the $j=0, v=1$ manifold, which borrow their intensity from the $j= \pm 1, v=0$ state owing to the Herzberg-Teller effect. This effect is pronounced for low disorder, but quickly smears out from $\sigma_{\phi}>0.2 \mathrm{rad}$. We do not observe appreciable intensity from the $j= \pm 1, v=1$ transitions for the ordered ring, because as stated above, it is not practical to compute enough states. We think that the lack of a vibronic progression (because 35 states were computed), as well as averaging over only 40 realizations of the disorder, explains the differences between Fig. 10 and Fig. 12 for high disorder. 
The emission spectra for high disorder show the same features as the Condon approximation results, however for the low-disorder limit we see the expected deviation due to the Herzberg-Teller effect. Most notably, the spectrum for an ordered system does not vanish, but shows the 0-1 emission transition arising from the Herzberg-Teller effect. For increasing disorder this effect vanishes, but for small disorder we see a reversal in the $I_{00} / I_{01}$ intensity ratio with $I_{01}$ being more intense (reflecting the results shown in Fig. 8). The HerzbergTeller correction has a significant effect on the emission spectra up to disorder comparable to room temperature, before being smeared by the usual vibronic progression. We also find a more accurate reproduction of the intensity ratio of the two peaks in the emission spectrum. The low-energy peak is still significantly less intense, but it is a significant improvement on the Condon approximation.

\section{SUMMARY AND CONCLUDING REMARKS}

This paper describes the optical properties of $\pi$-conjugated macrocycles. Since the lowenergy excitations of these systems are Frenkel excitons that couple to high-frequency dispersionless phonons, we employed the quantized Frenkel-Holstein model solved via the DMRG method to determine these optical properties.

First we considered optical emission from perfectly circular systems. Owing to optical selection rules, such systems radiate via two mechanisms: (i) within the Condon approximation, by thermally induced emission from the optically allowed $j= \pm 1$ states and (ii) beyond the Condon approximation, by emission from the $j=0$ state via coupling with a totally non-symmetric phonon (namely, the Herzberg-Teller effect). Using perturbation theory, we derived an expression for the Herzberg-Teller correction and showed via DMRG calculations that this expression soon fails as $\hbar \omega / J$ and the size of the macrocycle are increased.

Next, we considered the role of broken symmetry caused by static torsional disorder. In this case the quantum number $j$ no longer labels eigenstates of definite angular momentum, but instead labels localized local exciton groundstates (LEGSs) or quasi-extended states (QEESs). As noted for linear polymers, LEGSs define chromophores, with the higher energy QEESs being extended over numerous LEGSs. Within the Condon approximation (i.e., neglecting Herzberg-Teller corrections) we showed that increased disorder increases the emissive optical intensity, because all the LEGSs are optically active. Indeed, for large 
macrocycles with sufficiently large disorder the symmetry between optical absorption and emission is largely restored. There is still some asymmetry between absorption and emission, however, because although QEESs participate in the absorption intensity, Kasha's law implies ultra-fast relaxation from QEESs to LEGSs, whereby only LEGSs are emissive states. This relaxation from QEESs to LEGSs is the cause of ultra-fast 'dynamical' localization and anisotropic depolarization.

We next considered the combined role of broken symmetry and curvature, namely we explicitly evaluated the Herzberg-Teller correction via the DMRG method. The HerzbergTeller correction is most evident in the emission intensity ratio, $I_{00} / I_{01}$. In the Condon approximation $I_{00} / I_{01}$ is a constant function of curvature, whereas in practice it vanishes for closed rings and only approaches a constant in the limit of vanishing curvature. ${ }^{32}$

We calculated the optical spectra of cyclo-poly(para-phenylene ethynylene) for different amounts of torsional disorder within and beyond the Condon approximation. We showed how broken symmetry and the Herzberg-Teller effect explain the spectral features. We noted that the Herzberg-Teller correction to the $0-1$ emission vibronic peak is always significant.

Finally, we noted the qualitative similarities between the optical properties of conformationally disordered linear polymers and macrocycles in the limit of sufficiently large disorder, because in both cases they are determined by the optical properties of curved chromophores.

We conclude by returning to the issues of broken symmetry and localization. Some explanations of ultra-fast fluorescence depolarization in macrocycles in the literature assume that an initially excited delocalized state becomes localized via self-trapping by vibrational modes before emitting. As discussed in the Introduction, we do not think that the selftrapping is the dominant cause of exciton localization. Instead, we think that torsional disorder Anderson localizes the exciton center-of-mass wavefunction, with higher-energy states (QEESs) being more delocalized than lower-energy states (LEGSs). Thus, as in linear polymers, the energetic relaxation of QEESs to LEGSs explains ultra-fast fluorescence depolarization ${ }^{55}$.

Acknowledgments M. M. thanks Magdalen College for financial support through a Zvi and Ofra Meitar Magdalen Graduate Scholarship. 
Corresponding authors Email addresses: max.marcus@chem.ox.ac.uk and william.barford@chem.ox.ac.uk

APPENDIX A: DERIVATION OF AN EXPRESSION FOR THE HERZBERGTELLER EFFECT IN MACROCYCLES

The transition dipole moment between the electronic ground state, $|\mathrm{GS}, v\rangle$, and an excited state, $\left|\mathrm{EX}, v^{\prime}\right\rangle$, in the vibrational levels $v$ and $v^{\prime}$ associated with a normal coordinate, $Q^{(k)}$ is

$$
\mu_{v v^{\prime}} \equiv\left\langle\mathrm{GS}, v|\underline{\hat{\mu}}| \mathrm{EX}, v^{\prime}\right\rangle
$$

The Born-Oppenheimer approximation assumes that the full electronic-nuclear wavefunctions may be factorized as,

$$
|\mathrm{GS}, v\rangle \equiv \Psi_{\mathrm{GS}}\left(r, Q^{(k)}\right) \phi_{v}^{\mathrm{GS}}\left(Q^{(k)}\right)
$$

and

$$
\left|\mathrm{EX}, v^{\prime}\right\rangle \equiv \Psi_{\mathrm{EX}}\left(r, Q^{(k)}\right) \phi_{v^{\prime}}^{\mathrm{EX}}\left(Q^{(k)}\right)
$$

where the electronic wavefunction, $\Psi\left(r, Q^{(k)}\right)$, is parameterized by $Q^{(k)}$, while $\phi_{v}\left(Q^{(k)}\right)$ is the associated vibrational wavefunction. Here $k$ is the wavenumber of the normal coordinate.

Thus, using Eq. (A2) and Eq. (A3), Eq. (A1) becomes

$$
\mu_{v v^{\prime}}=\int \mu_{e}\left(Q^{(k)}\right) \phi_{v}^{\mathrm{GS}}\left(Q^{(k)}\right) \phi_{v^{\prime}}^{\mathrm{EX}}\left(Q^{(k)}\right) \mathrm{d} Q^{(k)}
$$

where

$$
\mu_{e}\left(Q^{(k)}\right)=\int \Psi_{\mathrm{GS}}\left(r, Q^{(k)}\right) \hat{\mu} \Psi_{\mathrm{EX}}\left(r, Q^{(k)}\right) \mathrm{d} r
$$

is the electronic transition dipole moment.

Performing a Taylor expansion on $\mu_{e}\left(Q^{(k)}\right)$ we have

$$
\mu_{e}\left(Q^{(k)}\right)=\mu_{e}\left(Q_{0}^{(k)}\right)+\left.\frac{\partial \mu_{e}}{\partial Q^{(k)}}\right|_{Q_{0}^{(k)}}\left(Q^{(k)}-Q_{0}^{(k)}\right)+\ldots
$$

which leads to

$$
\begin{aligned}
\mu_{v v^{\prime}} & =\mu_{e}\left(Q_{0}^{(k)}\right) \int \phi_{v}^{\mathrm{GS}}\left(Q^{(k)}\right) \phi_{v^{\prime}}^{\mathrm{EX}}\left(Q^{(k)}\right) \mathrm{d} Q^{(k)} \\
& +\left.\frac{\partial \mu_{e}}{\partial Q^{(k)}}\right|_{Q_{0}^{(k)}} \int \phi_{v}^{\mathrm{GS}}\left(Q^{(k)}\right)\left(Q^{(k)}-Q_{0}^{(k)}\right) \phi_{v^{\prime}}^{\mathrm{EX}}\left(Q^{(k)}\right) \mathrm{d} Q^{(k)} \\
& +\ldots
\end{aligned}
$$


Retaining only the first term on the right-hand-side of Eq. (A7) is the Condon approximation. If $\mu_{e}\left(Q_{0}^{(k)}\right)$ vanishes for symmetry reasons, as described in Section III, the second term - the Herzberg-Teller correction - is the leading-order term. The Herzberg-Teller term does not vanish for non-totally symmetric vibrations (i.e., excited levels of $k \neq 0$ modes).

We can further analyse the Herzberg-Teller term using perturbation theory. First, we consider

$$
\left.\frac{\partial \mu_{e}}{\partial Q^{(k)}}\right|_{Q_{0}^{(k)}}=\left.\frac{\partial}{\partial Q^{(k)}}\left(\int \Psi_{\mathrm{GS}}\left(\mathbf{r}, Q^{(k)}\right) \underline{\hat{\mu}} \Psi_{\mathrm{EX}}\left(\mathbf{r}, Q^{(k)}\right) \mathrm{d} \mathbf{r}\right)\right|_{Q_{0}^{(k)}} .
$$

Due to the large energy gap between the ground and first excited state, we will ignore any mixing of these states. However, the first excited electronic state $(j=0)$, which is totally symmetric, will acquire non-totally symmetric character by mixing with higher excited states. In particular, mixing with the $j= \pm 1$ states will render it dipole active. This mixing occurs via the exciton-phonon coupling term, which in the Frenkel-Holstein model is given by

$$
\hat{V}=-A \hbar \omega \sum_{n=1}^{N} Q_{n} \hat{a}_{n}^{\dagger} \hat{a}_{n}
$$

For cyclic systems, the local displacement, $Q_{n}$, is expressed as a Fourier expansion of the normal coordinates,

$$
Q_{n}=\sum_{k} Q^{(k)} \frac{e^{-i k n}}{\sqrt{N}}
$$

Considering only mixing between the $j=0$ and the $j= \pm 1$ states, we can express the perturbed wavefunction as,

$$
\Psi_{j=0}\left(Q^{(k)}\right)=\Psi_{j=0}+\sum_{j= \pm 1} \frac{\left\langle\Psi_{j=0}|\hat{V}| \Psi_{j}\right\rangle}{\Delta E_{0 j}} \Psi_{j}+\cdots
$$

Evaluating the matrix element in Eq. (A11) using Eq. (24) gives

$$
\left\langle\Psi_{j=0}|\hat{V}| \Psi_{j= \pm 1}\right\rangle=-\frac{A \hbar \omega}{\sqrt{N}} Q^{(k)}
$$

where only the $k= \pm 2 \pi / N$ terms in Eq. (A10) contribute. Thus, the perturbed $j=0$ wavefunction is

$$
\Psi_{j=0}\left(Q^{(k= \pm 2 \pi / N)}\right) \approx \Psi_{j=0}-\sum_{j= \pm 1} \frac{A \hbar \omega}{\sqrt{N} \Delta E_{0 j}} Q^{(k= \pm 2 \pi / N)} \Psi_{j} .
$$

Finally, using Eq. (20), the electronic transition dipole moment with the groundstate is

$$
\mu_{e}\left(Q^{(k)}\right)=-\frac{A \hbar \omega}{\sqrt{N} \Delta E_{0 j}} Q^{(k)} \times \frac{\mu_{0} \sqrt{N}}{2}
$$


for each spatial component.

The electronic contribution to the Herzberg-Teller term is thus

$$
\frac{\partial \mu_{e}}{\partial Q^{(k)}}=-\frac{\mu_{0} A \hbar \omega}{2 \Delta E_{0 j}}
$$

As discussed in Section III,

$$
\Delta E_{0 j}=2 J\left(1-\cos \left(\frac{2 \pi}{N}\right)\right) \approx J\left(\frac{2 \pi}{N}\right)^{2} ; \quad N \gg 1,
$$

and as $I_{01} \propto\left|\mu_{01}\right|^{2}$, we find that

$$
I_{01} \propto \mu_{0}^{2} A^{2}\left(\frac{\hbar \omega}{J}\right)^{2} \times N^{4} \times \mu_{\mathrm{vib}}^{2} .
$$

The vibrational contribution, $\mu_{\mathrm{vib}}$, is given by the matrix element

$$
\mu_{\mathrm{vib}}=\int \phi_{v}^{\mathrm{GS}}\left(Q^{(k)}\right)\left(Q^{(k)}-Q_{0}^{(k)}\right) \phi_{v^{\prime}}^{\mathrm{EX}}\left(Q^{(k)}\right) \mathrm{d} Q^{(k)} .
$$

Since $\left\langle\Psi_{j=0}\left|Q^{(k \neq 0)}\right| \Psi_{j=0}\right\rangle=0$, the $j=0$ excited state potential energy surface of a nontotally symmetric normal coordinate is not displaced relative to the ground state potential energy surface ${ }^{56}$. Thus, the evaluation of Eq. (A18) gives

$$
\mu_{\mathrm{vib}}=(v / 2)^{1 / 2} \delta_{v, v^{\prime}+1}
$$

(recalling that $Q$ is the dimensionless normal coordinate). Finally, taking $v^{\prime}=0$ for the $j=0$ excited electronic state and $v=1$ for the electronic groundstate, we have the $0-1$ emission intensity from the $j=0$ state,

$$
I_{01} \propto N^{4} \mu_{0}^{2} A^{2}\left(\frac{\hbar \omega}{J}\right)^{2}
$$




\section{Comments}

- Equation (A20) is only valid to first order in perturbation theory and therefore we require

$$
\left|\frac{A \hbar \omega}{\sqrt{N}} Q^{(k)}\right| \ll \Delta E_{0 j},
$$

or

$$
\left(\frac{\hbar \omega}{J}\right) \frac{A}{N^{3 / 2}} \ll 1
$$

Departures from this prediction are shown in Fig. 3.

- Equation (A20) is valid to all orders of the Taylor expansion of $\mu_{e}\left(Q^{(k)}\right)$, because the same result is obtained by using Eq. (A14) directly in Eq. (A5).

- Equation (A20) also applies to the 0-1 absorption into the $j=0$ state, as discussed in Section VIB.

\section{APPENDIX B: THE ELECTRONIC TRANSITION DIPOLE MOMENT OF A CURVED CHROMOPHORE IN THE CONDON APPROXIMATION}

In the Condon approximation the electronic transition dipole moment is

$$
\underline{\mu}=\sum_{n=1} \underline{\mu}_{n} \Psi(n)
$$

where $\underline{\mu}_{n}$ is the transition dipole moment on monomer $n$ and $\Psi(n)$ is the exciton center-ofmass wavefunction.

$$
\underline{\mu}_{n}=\mu_{0} \underline{\hat{r}}_{n}
$$

where $\underline{\hat{r}}_{n}$ is the unit vector denoting the orientation of the $n$th monomer and $\mu_{0}$ is the transition dipole moment of a single monomer. To a good approximation, for a LEGS we may assume a plane-wave center-of-mass wavefunction, i.e., $\Psi(n)=1 / \sqrt{\mathrm{CL}}$, where CL is the conjugation length (or chromophore size) in units of the monomer length. Then,

$$
\underline{\mu}_{j}(N)=\frac{\mu_{0}}{\sqrt{\mathrm{CL}}} \sum_{n \in \mathrm{CL}} \underline{\hat{r}}_{n}
$$

where the sum is over all monomers spanned by the LEGS (i.e., the chromophore). Equation (B3) indicates that in the Condon approximation the electronic transition dipole moment is 
the coherent sum of the monomeric transition dipole moments, where

$$
\underline{L}=\sum_{n \in \mathrm{CL}} \underline{\hat{r}}_{n}
$$

is the chord (in units of the monomer length) spanning the chromophore, as shown in Fig. 7. Thus, the square of the transition dipole moment is

$$
|\mu|^{2}=\mu_{0}^{2}\left(\frac{L^{2}}{\mathrm{CL}}\right)
$$

For $\theta \leq \pi$ (as defined in Fig. 7), $L=2 R \sin (\theta / 2)$ (with $R$, the radius, is in units of the monomer length) and thus using $\mathrm{CL}=R \theta$,

$$
|\mu|^{2}=4 \mu_{0}^{2}\left(\frac{R \sin ^{2}(\theta / 2)}{\theta}\right) .
$$

For $\theta \ll 1$ Eq. (B6) becomes,

$$
|\mu|^{2}=\mu_{0}^{2} \mathrm{CL}\left(1-\theta^{2} / 12+\cdots\right)
$$

showing that curvature reduces the oscillator strength for an almost straight chromophore.

Likewise, for $\theta>\pi$,

$$
|\mu|^{2}=4 \mu_{0}^{2}\left(\frac{R \sin ^{2}(\pi-\theta / 2)}{\theta}\right)
$$

and for $(2 \pi-\theta) \ll 1$

$$
|\mu|^{2}=\mu_{0}^{2} \frac{(2 \pi R-\mathrm{CL})^{2}}{\mathrm{CL}}
$$

showing that the oscillator strength vanishes if the chromophore spans the ring.

\section{APPENDIX C: CURVED CHROMOPHORES AND THE HERZBERG-TELLER} EFFECT

We write the transition dipole moment from the zeroth vibrational level of the lowest excited electronic state to the first vibrational level of the electronic ground state as

$$
\mu_{01}=\mu_{01}^{\mathrm{C}}+\mu_{01}^{\mathrm{HT}}
$$

where $\mu_{01}^{\mathrm{C}}$ is the Condon contribution (given by the first term on the right-hand side of Eq. (A7)), while $\mu_{01}^{\mathrm{HT}}$ is the Herzberg-Teller correction to the Condon term. Since $I_{01} \propto \mu_{01}^{2}$,

$$
I_{01}=I_{01}^{\mathrm{C}}+2\left(I_{01}^{\mathrm{C}} I_{01}^{\mathrm{HT}}\right)^{1 / 2}+I_{01}^{\mathrm{HT}} .
$$


As shown previously, ${ }^{33,43}$ in the Condon approximation the $I_{00} / I_{01}^{\mathrm{C}}$ emission intensity ratio is

$$
\frac{I_{00}}{I_{01}^{\mathrm{C}}}=\frac{\mathrm{IPR}}{S(1)},
$$

where the inverse participation ratio, IPR, is defined in Eq. (12) and $S(1)$ is the HuangRhys parameter for a single monomer. Since $I_{00}$ arises from the coherent sum of monomer transition dipole moments (i.e., $I_{00} \equiv I_{00}^{\mathrm{C}}$; see Appendix A and Appendix B), which tends to vanish as the chromophore becomes more curved, Eq. (C2) and Eq. (C3) indicate that curvature and Herzberg-Teller corrections determine $I_{01}$.

Now, if the LEGS wavefunction is uniformly distributed over part of the macrocycle spanned by $\theta$, then its associated optical intensity, being proportional to the square of transition dipole moment, is given by the expressions in Appendix B. Moreover, IPR is proportional to the conjugation length, $\mathrm{CL}$, where $\mathrm{CL}=R \theta$. Thus, using Eq. (C3), Eq. (B6) or Eq. (B8), and rearranging Eq. (C2) we find,

$$
\frac{I_{00}}{I_{01} \mathrm{IPR}}=\frac{1}{\left(S(1)+2(S(1) B)^{1 / 2} \frac{\theta}{2 \sin (\theta / 2)}+B\left(\frac{\theta}{2 \sin (\theta / 2)}\right)^{2}\right)}
$$

for $0 \leq \theta \leq \pi$ and

$$
\frac{I_{00}}{I_{01} \mathrm{IPR}}=\frac{1}{\left(S(1)+2(S(1) B)^{1 / 2} \frac{\theta}{2 \sin (\pi-\theta / 2)}+B\left(\frac{\theta}{2 \sin (\pi-\theta / 2)}\right)^{2}\right)}
$$

for $\pi<\theta \leq 2 \pi$, where $B \propto I_{01}^{\mathrm{HT}}$. When the LEGSs almost span the ring, i.e., for $(2 \pi-\theta) \ll 1$, Eq. (C5) becomes

$$
\frac{I_{00}}{I_{01} \mathrm{IPR}}=\frac{1}{\left(S(1)+2(S(1) B)^{1 / 2} \frac{\theta}{(2 \pi-\theta)}+B\left(\frac{\theta}{(2 \pi-\theta)}\right)^{2}\right)},
$$

which evidently vanishes when $\theta=2 \pi$.

Equation (C5), derived in the adiabatic (Born-Oppenheimer) limit, is in qualitative agreement with Eq. (24) of Hestand and Spano ${ }^{32}$, derived in the anti-adiabatic limit. As shown in Section III B, in the anti-adiabatic limit the Herzberg-Teller term, $B$, becomes independent of $\hbar \omega / J$, making the agreement with ef $^{32}$ more quantitative.

1 H. Shirakawa, E. J. Louis, A. G. Macdiarmid, C.K. Chiang, and A. J. Heeger, J. Chem. Soc., Chem. Commun., 578 (1977). 
2 A. J. Heeger, S. Kivelson, J. R. Schrieffer, and W. P. Su, Rev. Mod. Phys., 60, 781 (1988).

3 A. O. Patil, A. J. Heeger, and F. Wudl, Chem. Rev., 88, 183 (1988).

4 J. H. Burroughes, D. D. C. Bradley, A. R. Brown, R. N. Marks, K. Mackay, R. H. Friend, P. L. Burns, and A. B. Holmes, Nature, 347, 539 (1990).

5 R. H. Mitchell, Eur. J. Org. Chem., 2695 (1999).

6 M. Iyoda, M. Hasegawe, and Y. Miyake, Chem. Rev., 104, 5085 (2004).

7 J. S. Kim and D. T. Quang, Chem. Rev., 107, 3780 (2007).

8 M. Iyoda, J. Yamakawa, and M. J. Rahman, Angew. Chem. Int. Ed., 50, 10522 (2011).

9 R. Herges, Nature, 450, 36 (2007).

10 R. Herges, Chem. Rev., 106, 4820 (2006).

11 H. Meier, Synthesis, 1213 (2002).

12 K. S. Kim, J. M Lim, A. Osuka, and D. Kim, J. Photochem. Photobiol. C, 9, 13 (2008).

13 J. K. Sprafke, D. V. Kondratuk, M. Wykes, A. L. Thompson, M. Hoffmann, R. Drevinskas, W.H. Chen, C. K. Yong, J. Kärnbratt, J. E. Bullock, M. Malfois, M. R. Wasielwski, B. Albinsson, L. M. Herz, D. Zigmantas, D. Beljonne, and H. L. Anderson, J. Am. Chem. Soc., 133, 17262 (2011).

14 C.-K. Yong, P. Parkinson, D. V. Kondratuk, W.-H. Chen, A. Stannard, A. Summerfield, J. K. Sprafke, M. C. O’Sullivan, P. H. Beton, H. L. Anderson, and L. M. Herz, Chem. Sci., 6, 181 (2015).

15 C. Camacho, T. A. Niehaus, K. Itami, and S. Irle, Chem. Sci., 4, 187 (2013).

16 L. Adamska, I. Nayyar, H. Chen, A. K. Swan, N. Oldani, S. Fernandez-Alberti, M. R. Golder, R. Jasti, S. K. Doorn, and S. Tretiak, Nano Lett., 14, 6539 (2014).

17 A. V. Aggarwal, A. Thiessen, A. Idelson, D. Kalle, D. Würsch, T. Stangl, F. Steiner, S.-S. Jester, J. Vogelsang, S. Höger, and J. M. Lupton, Nature Chemistry, 5, 964 (2013).

18 A. Thiessen, D. Würsch, S.-S. Jester, A. V. Aggarwal, S. Bange, J. Vogelsang, S. Höger, and J. M. Lupton, J. Phys. Chem. B 119, 9949 (2015).

19 B. M. Wong, J. Phys. Chem. C, 113, 21921 (2009).

20 J. Yang, S. Ham, T.-W. Kim, K. H. Park, K. Nakao, H. Shimizo, M. Iyoda, and D. Kim, J. Phys. Chem. B, 119, 4116 (2015).

21 K. Becker, P. G. Lagoudakis, G. Gaefke, S. Höger and J. M. Lupton, Angew. Chem. Int. Ed., 46, 3450 (2007). 
22 O. Mongin, A. Schuwey, M.-A. Vallot and A. Gossauer, Tetrahedron Letters, 40, 8347 (1999).

23 B. D. Steinberg and L. T. Scott, Angew. Chem. Int. Ed., 48, 5400 (2009).

24 W. Barford and D. Trembath, Phys. Rev. B, 80, 12 (2009).

25 D. V. Makhov and W. Barford, Phys. Rev. B, 81, 165201 (2010).

26 L. D. Landau, Phys. Z. Sowjetunion, 3, 884 (1933).

27 D. K. Campbell, A. R. Bishop, and K. Fesser, Phys. Rev. B, 26, 6862 (1982).

28 O. R. Tozer and W. Barford, Phys. Rev. B, 89, 155434, (2014).

29 W. Barford, M. Marcus, and O. R. Tozer, J. Phys. Chem. A 120, 615 (2016).

30 P. W. Anderson, Phys. Rev., 109, 1492 (1958).

31 N. F. Mott and W. D. Twose, Adv. Phys., 10, 107 (1961).

32 N. J. Hestand and F. C. Spano, J. Phys. Chem. B, 118, 8352 (2014).

33 M. Marcus, O. Tozer, and W. Barford, J. Chem. Phys, 141, 164102 (2014).

34 J. M. Lupton, ChemPhysChem, 13, 901 (2012).

35 F. C. Spano and H. Yamagata, J. Phys. Chem. B, 115, 5133 (2011).

36 F. C. Spano, Acc. Chem. Res., 43, 429 (2010).

37 H. Yamagata and F. C. Spano, J. Chem. Phys., 135, 054906 (2011).

38 H. Yamagata, N. J. Hestand, F. C. Spano, A. Köhler, C. Scharsich, S. T. Hoffmann, and H. Bässler, J. Chem. Phys., 139, 114903 (2013).

39 H. Yamagata and F. C. Spano, Phys. Chem. Lett., 5, 622 (2014).

40 F. Paquin, H. Yamagata, N. J. Hestand, M. Sakowicz, N. Bérubé, M. Côte, L. X. Reynolds, S. A. Haque, N. Stingelin, F. C. Spano, and C. Silva, Phys. Rev. B, 88, 155202 (2013).

41 W. Barford, J. Phys. Chem. A, 117, 2665 (2013).

42 O. R. Tozer and W. Barford, J. Phys. Chem. A, 116, 10310 (2012).

43 W. Barford and M. Marcus, J. Chem. Phys, 141, 164101 (2014).

44 S. R. White, Phys. Rev. Lett., 69, 2863 (1992).

45 S. R. White, Phys. Rev. B, 48, 10345 (1993).

46 C. Zhang, E. Jeckelmann, and S. R. White, Phys. Rev. Lett., 80, 2661 (1998).

47 The prediction $I_{00} / I_{01}=\mathrm{IPR} / S(1)$ was derived by Spano and Yamagata ${ }^{35,37}$ for linear systems for all parameter ranges. The prediction that $I_{00} / I_{01} \simeq A_{00} / A_{01} \simeq \mathrm{IPR} / S(1)$ was derived by Barford and Marcus ${ }^{43}$ and assumed the Born-Oppenheimer-Condon approximation.

48 G. Herzberg and E. Teller, Z. Phys. Chem., Abt. B, 21, 410 (1933). 
49 The Born-Oppenheimer factorization of the excitonic and vibrational wavefunctions is also valid if the condition in Eq. (28) is satisfied.

50 B. Kramer and A. MacKinnon, Rep. Prog. Phys., 56, 1469 (1993).

51 A. V. Malyshev and V. A. Malyshev, Phys. Rev. B, 63, 195111 (2001).

52 LEGSs are defined by the condition $\left|\sum_{n} \Psi_{j}(n)\right| \Psi_{j}(n) \| \gtrsim 0.95 .{ }^{51}$

53 W. Barford, I. Boczarow, and T. Wharram, J. Phys. Chem. A, 115, 111 (2011)

54 T. Kawase, Synlett, 17, 2609 (2007).

55 For other causes of fluoresence depolarization, see the Appendix in, W. Barford and O. R. Tozer, J. Chem. Phys, 141, 164103 (2014).

56 See, for example, F. Negri and G. Orlandi in Computational Photochemistry, ed. M. Olivucci, Elsevier, Amsterdam (2005). 


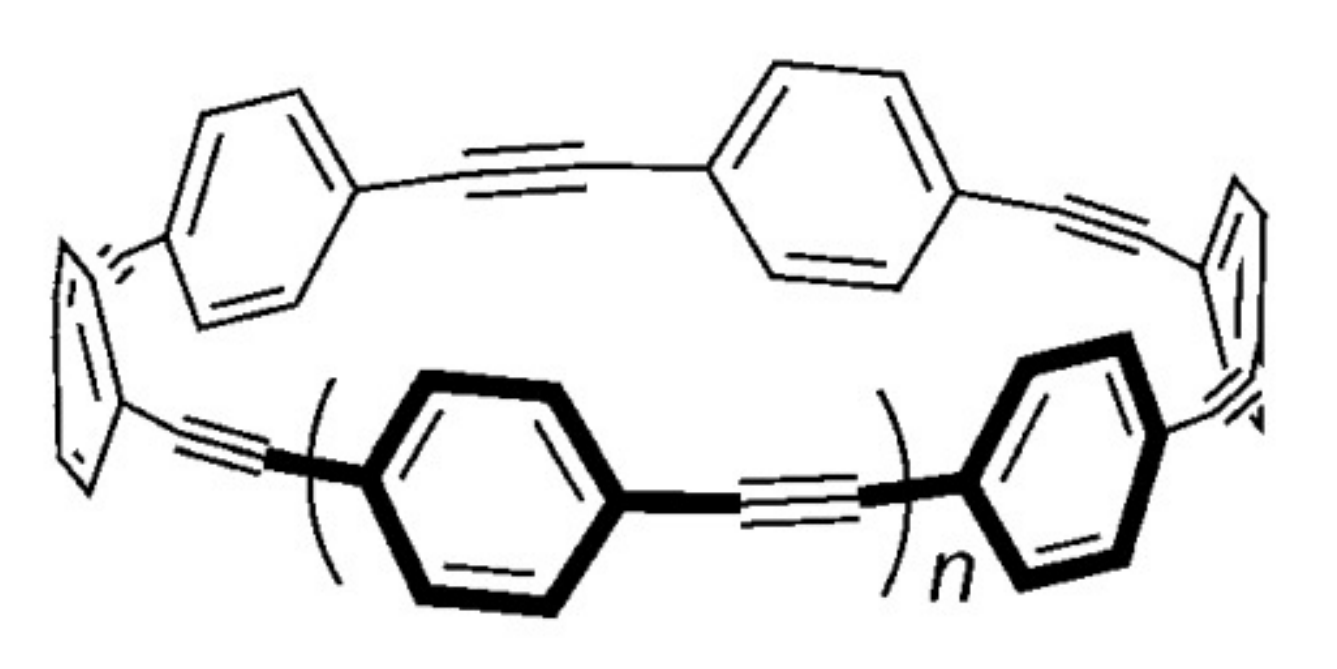




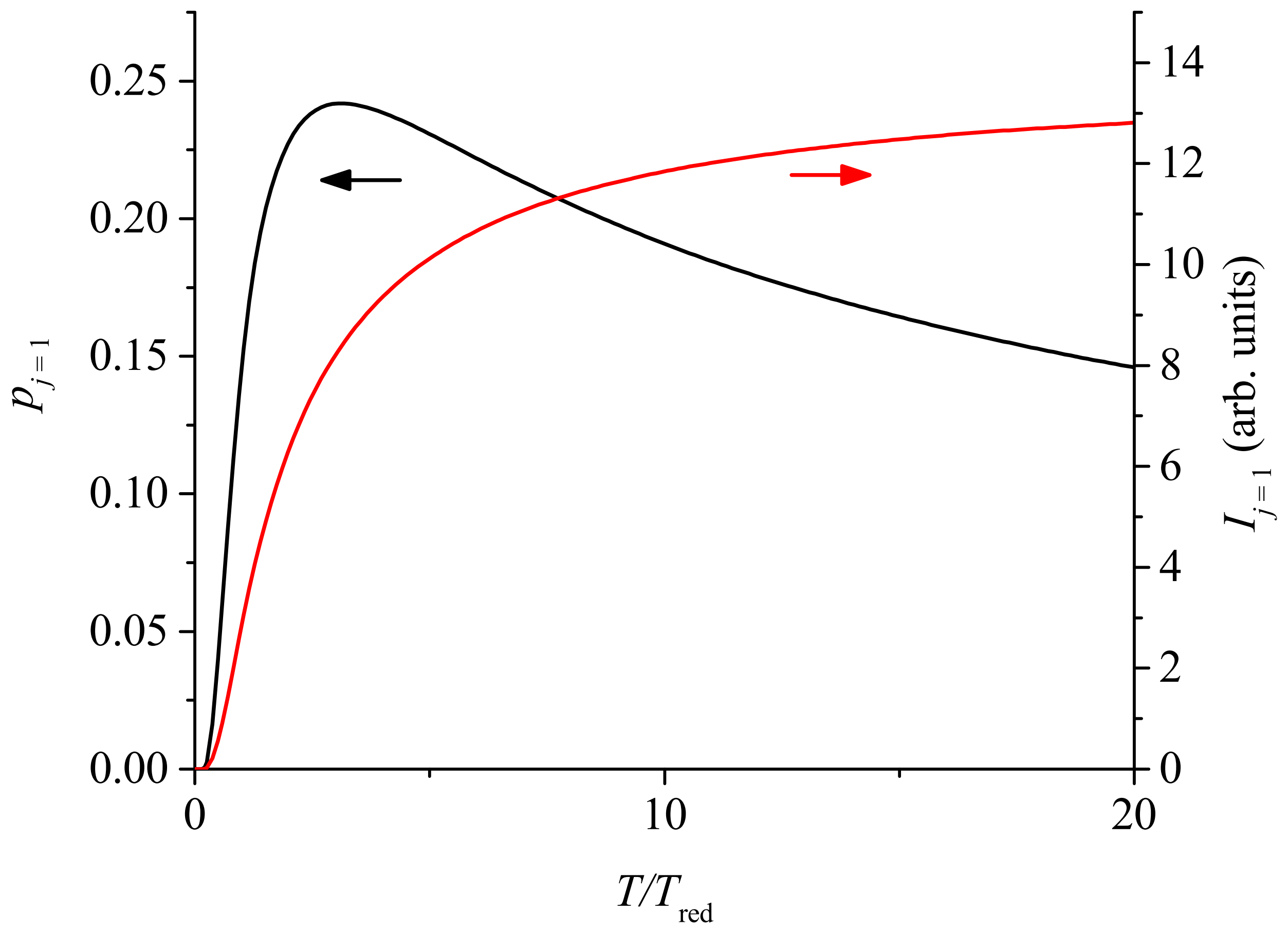



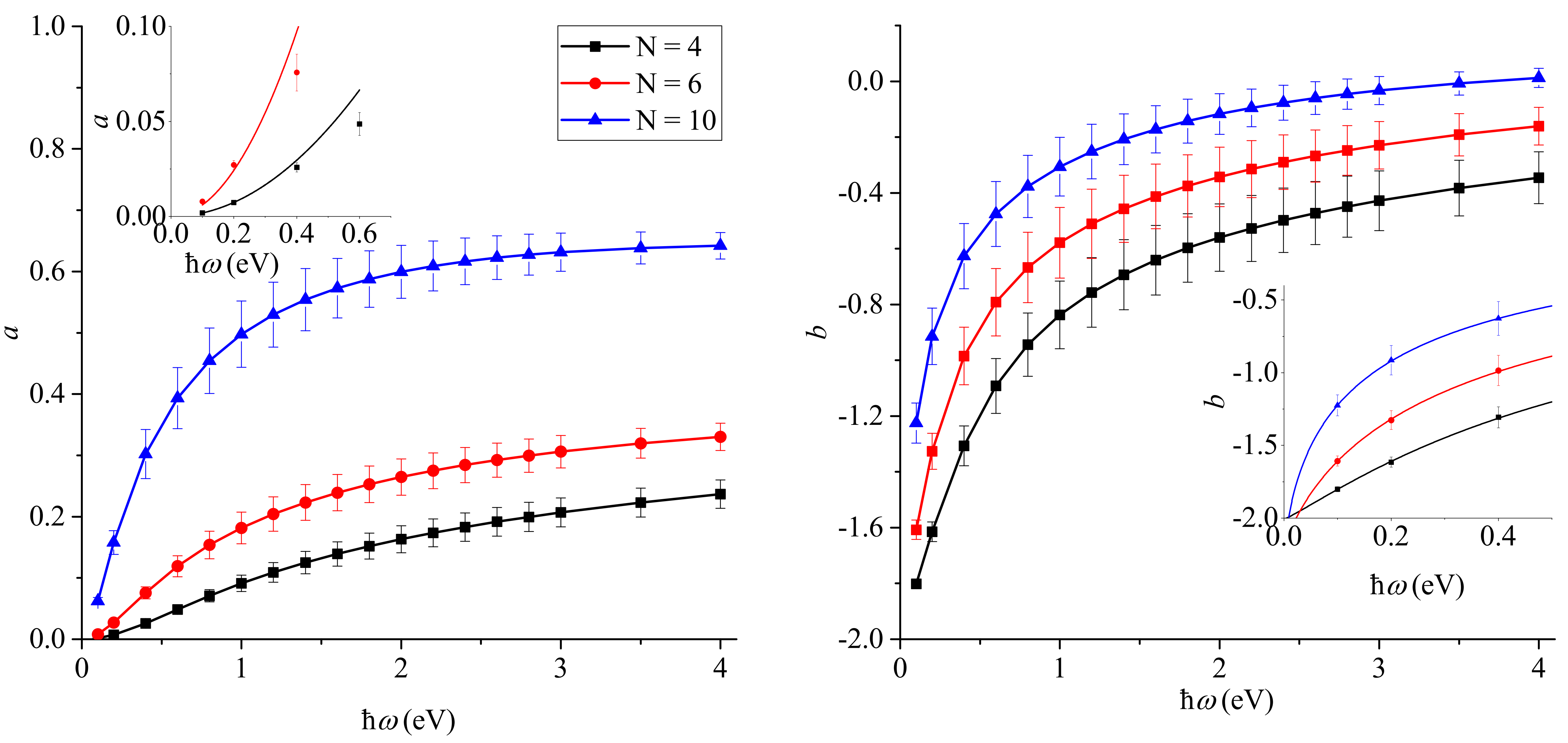


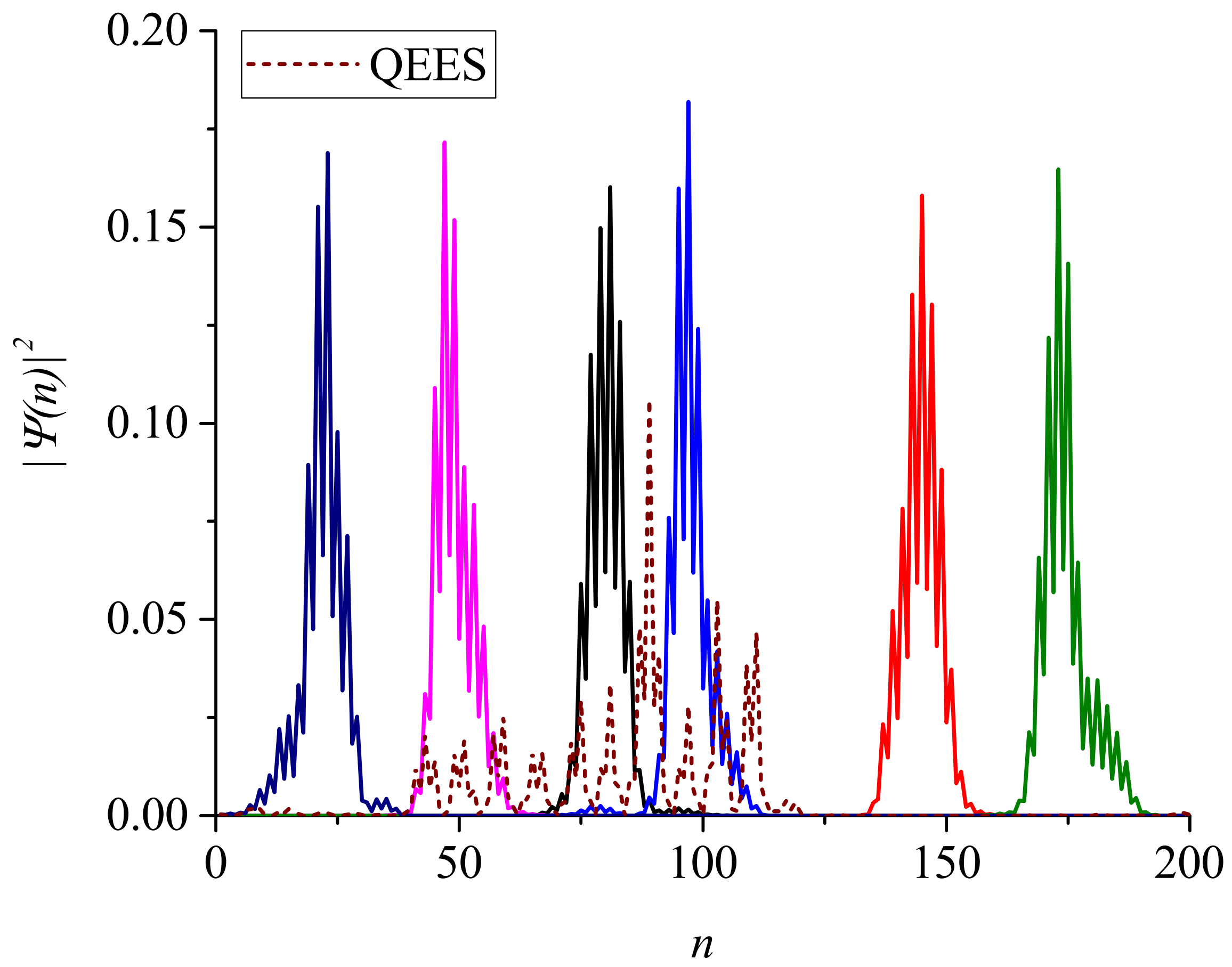




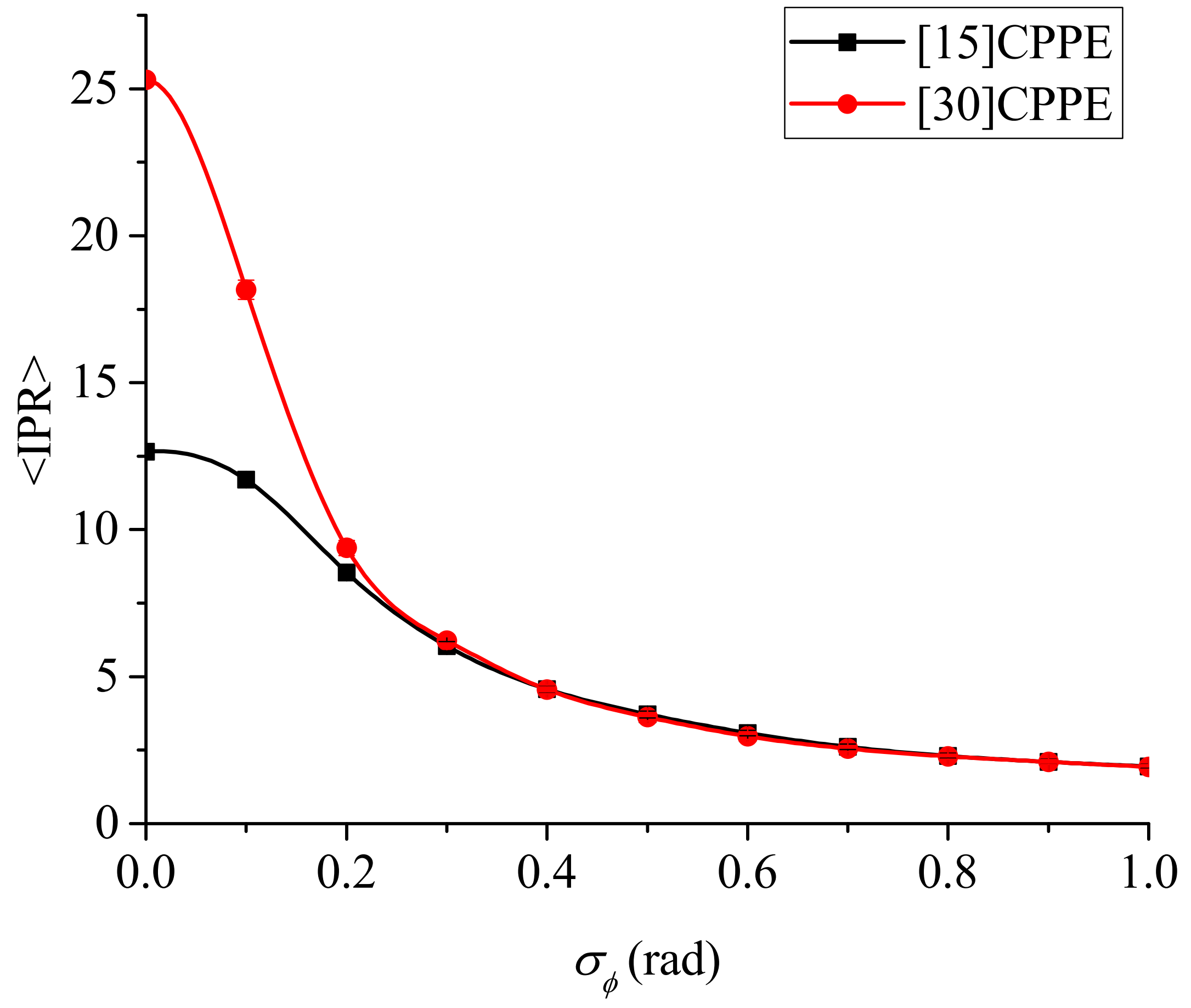




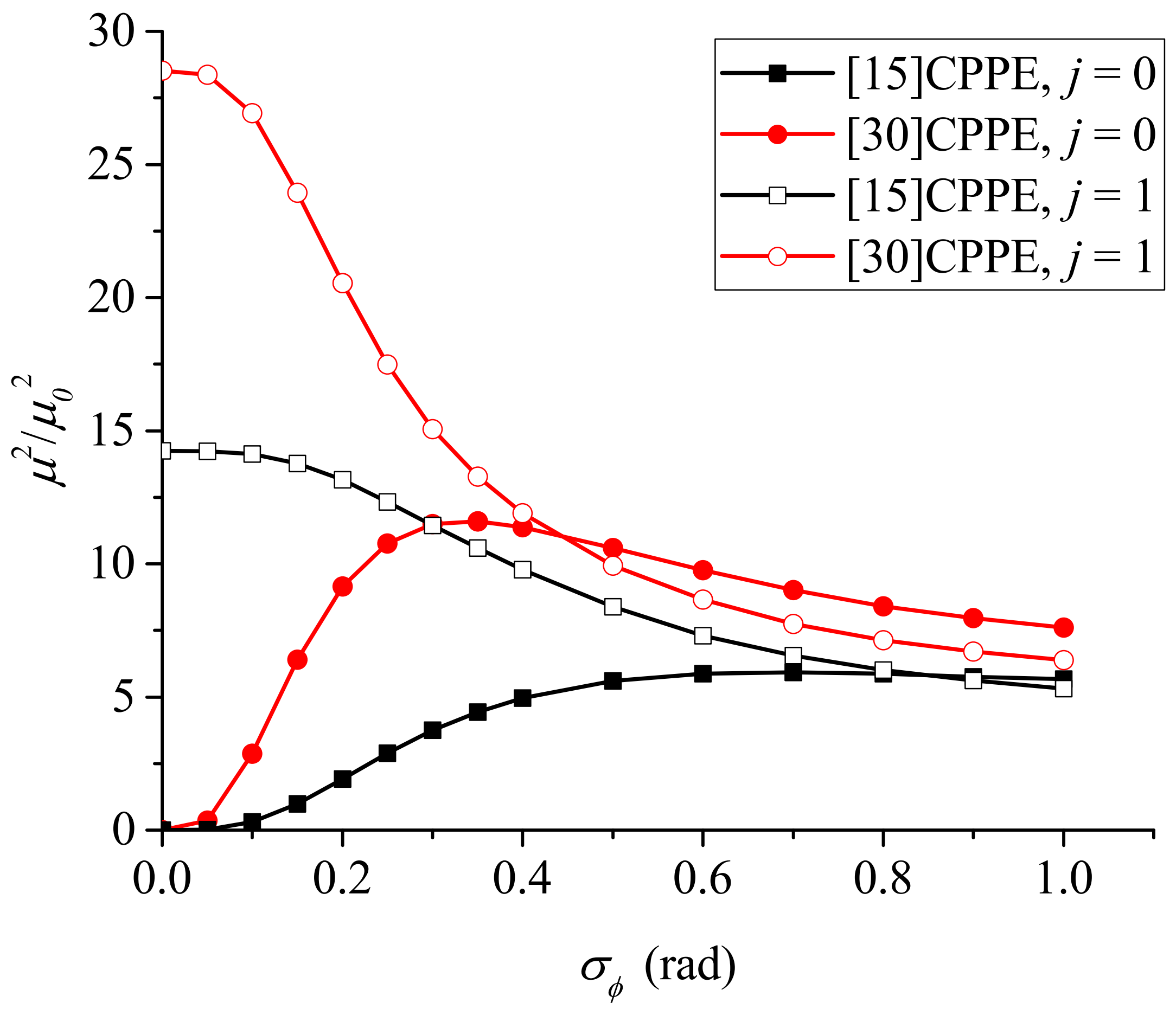




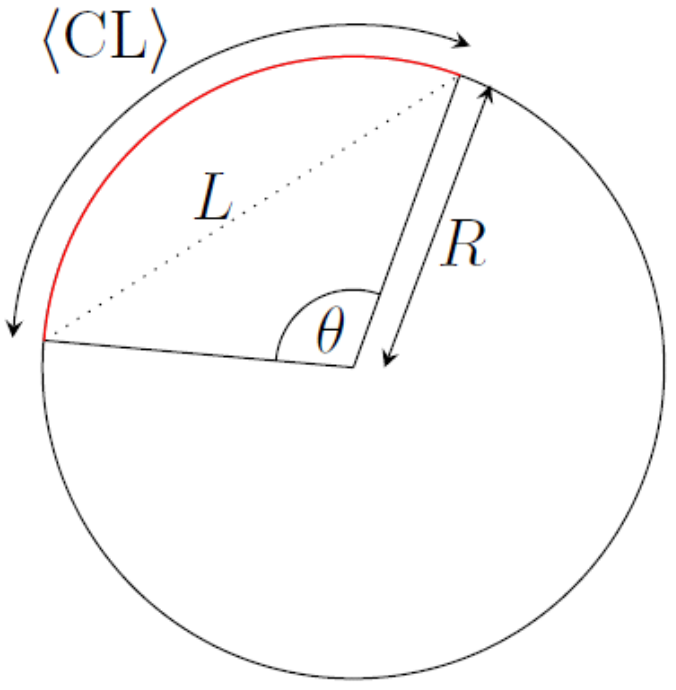




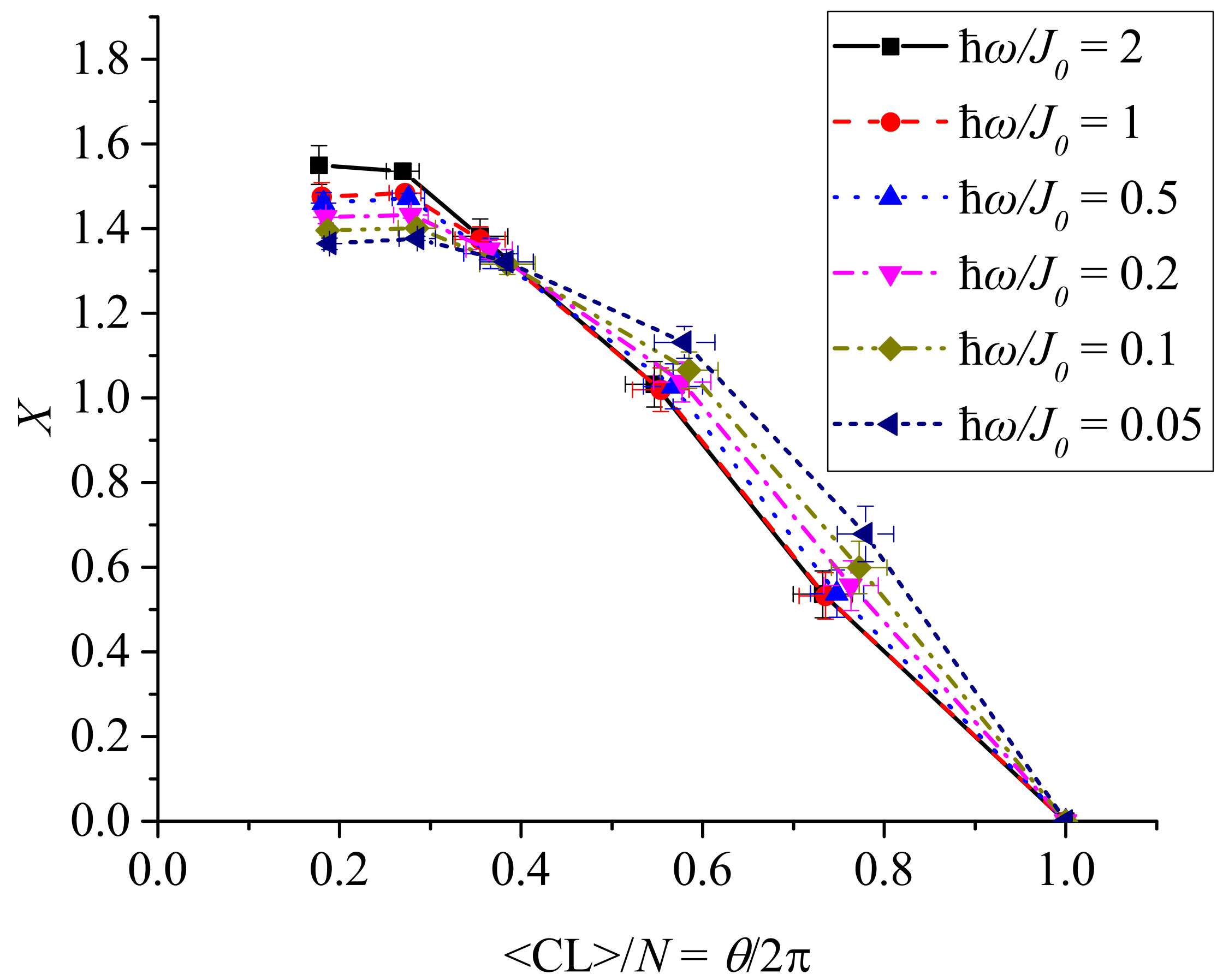




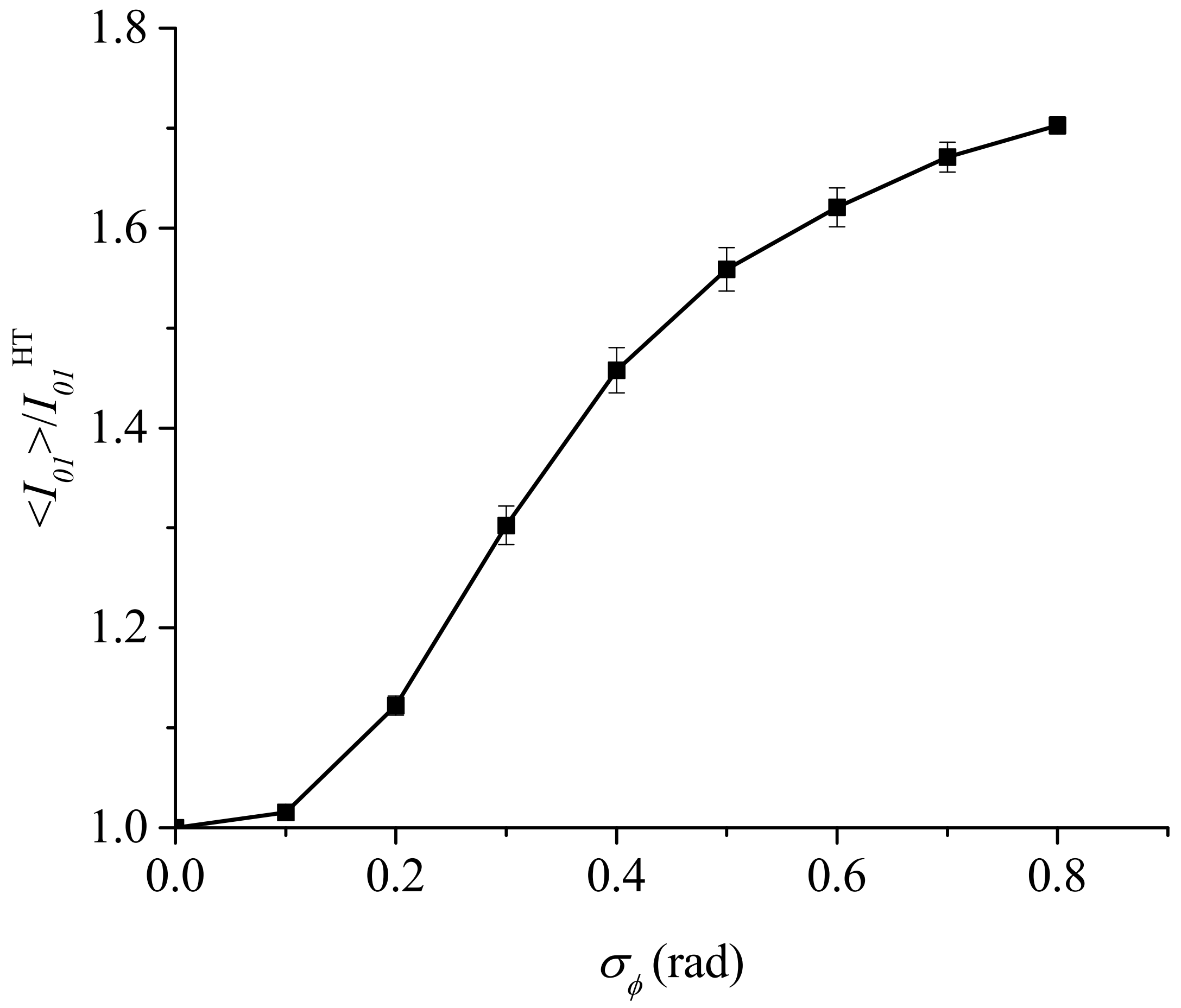




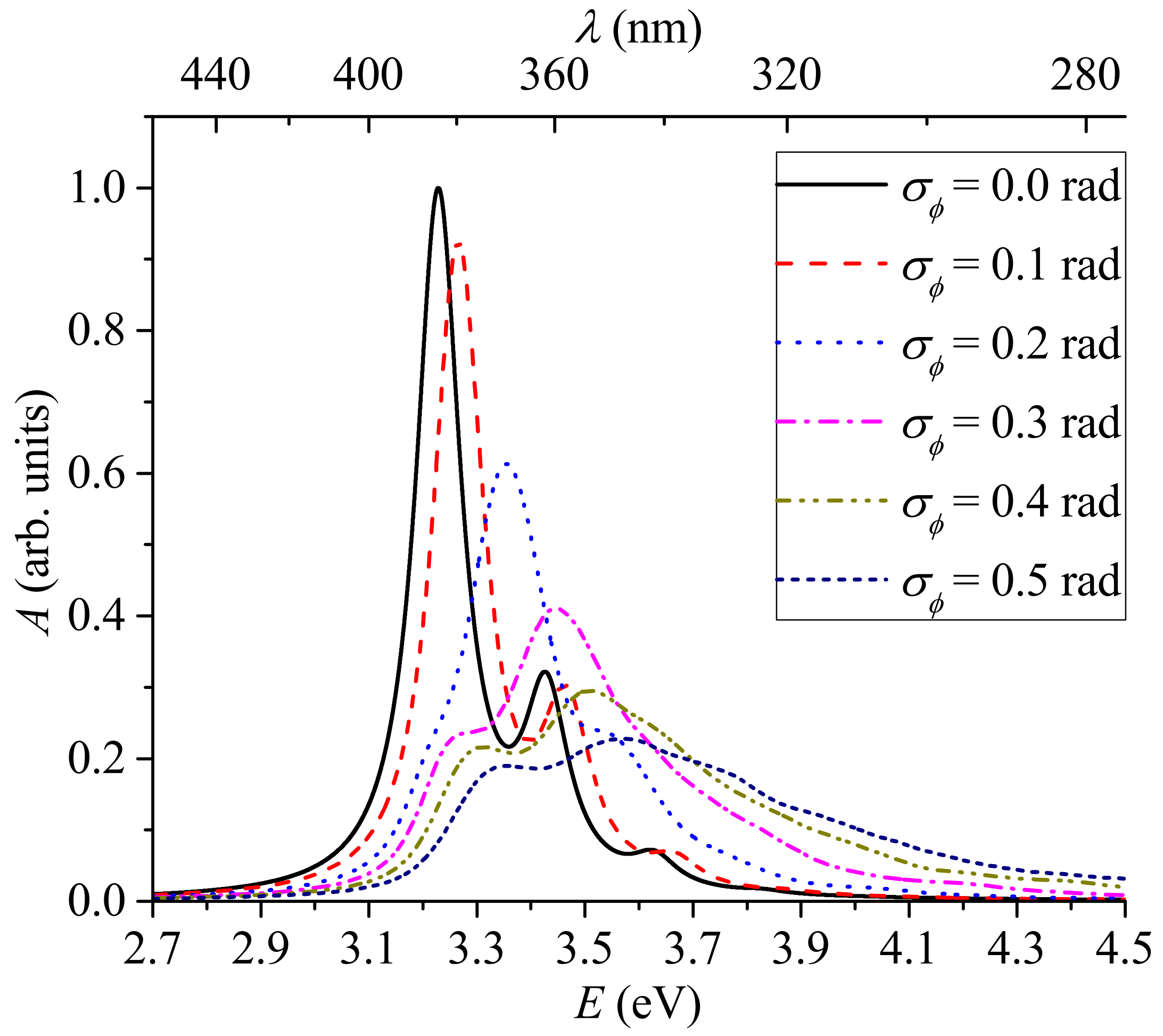




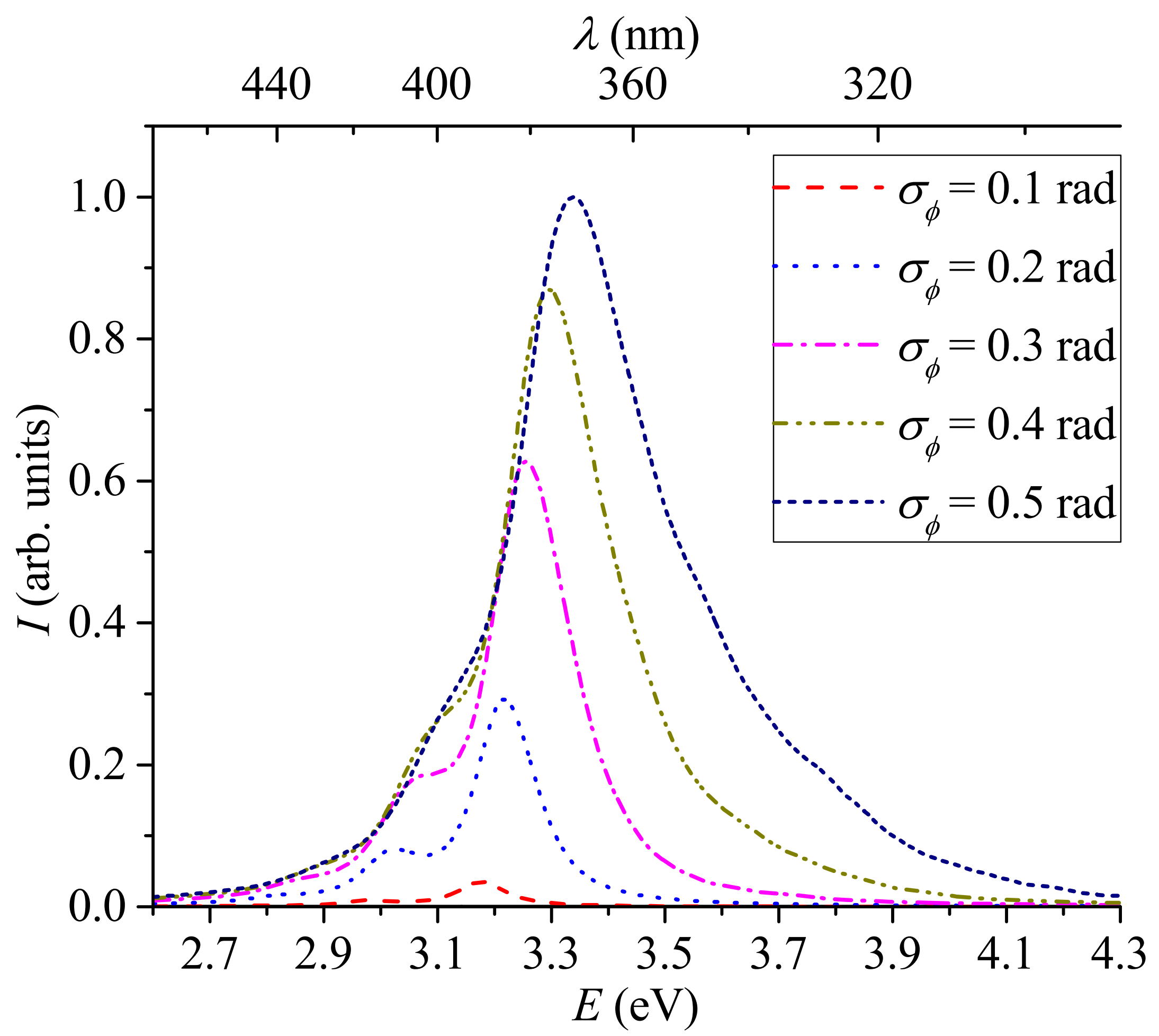




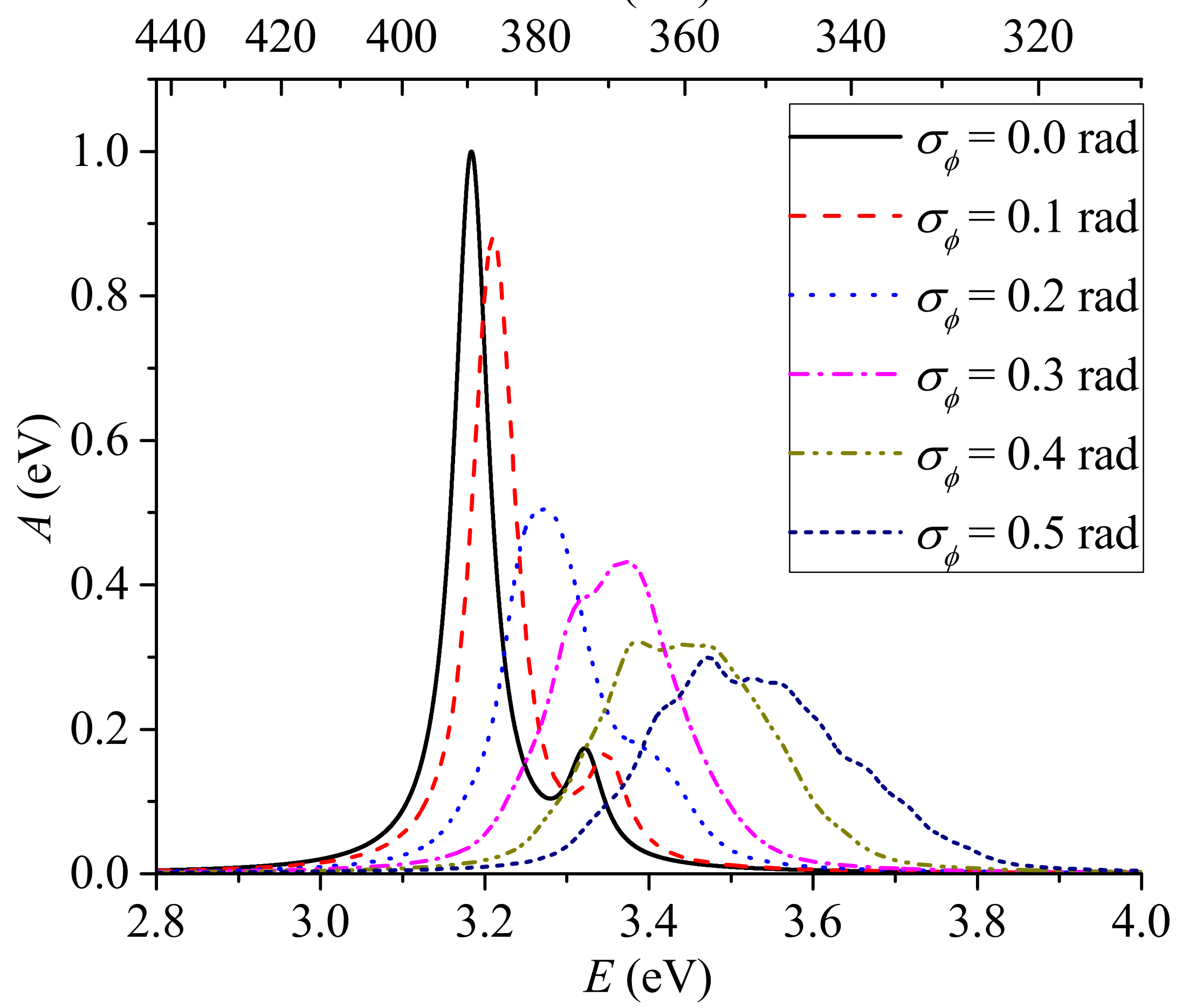


